\title{
Fine Structure and Spin Dynamics of Linearly Polarized Indirect Excitons in Two- Dimensional CdSe/CdTe Colloidal Heterostructures
}

Raj Pandya, ${ }^{\dagger}$ Violette Steinmetz, ${ }^{\ddagger}$ Yuttapoom Puttisong, ${ }^{\S}$ Marion Dufour, ${ }^{\perp}$ (৫) Weimin M. Chen, ${ }^{\S}$

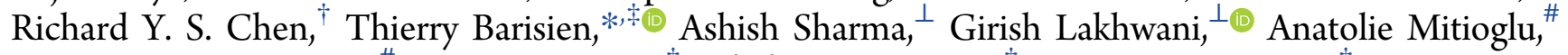
Peter C. M. Christianen, ${ }^{\#}$ Laurent Legrand, ${ }^{\ddagger}$ Frédérick Bernardot, ${ }^{\ddagger}$ Christophe Testelin, ${ }^{\ddagger}$ Alex W. Chin, ${ }^{\ddagger}$ Sandrine Ithurria, ${ }^{\| \odot}$ Maria Chamarro, ${ }^{\ddagger}$ and Akshay Rao ${ }^{\dagger}$

${ }^{\dagger}$ Cavendish Laboratory, University of Cambridge, J.J. Thomson Avenue, CB3 0HE Cambridge, United Kingdom

${ }^{\ddagger}$ Sorbonne Université CNRS-UMR 7588, Institut des NanoSciences de Paris, INSP, 4 place Jussieu, F-75005 Paris, France

${ }^{\S}$ Functional Electronic Materials, Department of Physics, Chemistry and Biology, Linköping University, 58183 Linköping, Sweden

"Laboratoire de Physique et d'Etude des Matériaux, ESPCI Paris, PSL Research University, CNRS, 10 rue Vauquelin, 75005 Paris, France

${ }^{\perp}$ ARC Centre of Excellence in Exciton Science, School of Chemistry, The University of Sydney, Sydney, New South Wales 2006, Australia

${ }^{\#}$ High Field Magnet Laboratory (HFML - EMFL), Radboud University, 6525 ED Nijmegen, The Netherlands

\section{Supporting Information}

ABSTRACT: Heterostructured two-dimensional colloidal nanoplatelets are a class of material that has attracted great interest for optoelectronic applications due to their high photoluminescence yield, atomically tunable thickness, and ultralow lasing thresholds. Of particular interest are laterally heterostructured core-crown nanoplatelets with a type-II band alignment, where the in-plane spatial separation of carriers leads to indirect (or charge transfer)
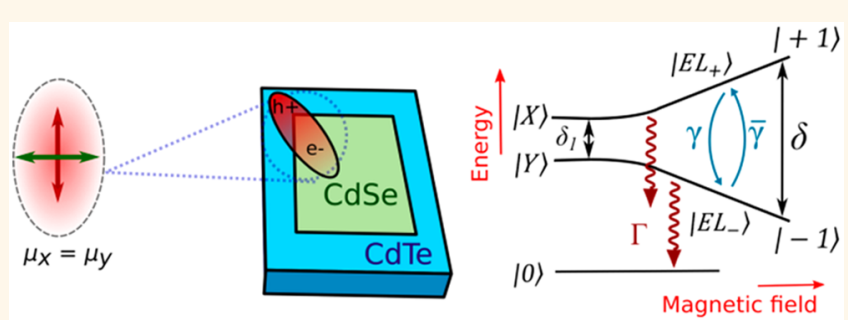
excitons with long lifetimes and bright, highly Stokes shifted emission. Despite this, little is known about the nature of the lowest energy exciton states responsible for emission in these materials. Here, using polarization-controlled, steady-state, and time-resolved photoluminescence measurements, at temperatures down to $1.6 \mathrm{~K}$ and magnetic fields up to $30 \mathrm{~T}$, we study the exciton fine structure and spin dynamics of archetypal type-II $\mathrm{CdSe} / \mathrm{CdTe}$ core-crown nanoplatelets. Complemented by theoretical modeling and zero-field quantum beat measurements, we find the bright-exciton fine structure consists of two linearly polarized states with a fine structure splitting $\sim 50 \mu \mathrm{eV}$ and an indirect exciton Landé $g$-factor of 0.7 . In addition, we show the exciton spin lifetime to be in the microsecond range with an unusual $B^{-3}$ magnetic field dependence. The discovery of linearly polarized exciton states and emission highlights the potential for use of such materials in display and imaging applications without polarization filters. Furthermore, the small exciton fine structure splitting and a long spin lifetime are fundamental advantages when envisaging $\mathrm{CdSe} / \mathrm{CdTe}$ nanoplatelets as elementary bricks for the next generation of quantum devices, particularly given their ease of fabrication.

KEYWORDS: CdSe/CdTe colloidal nanoplatelets, indirect excitons, high magnetic fields, core-crown heterostructure, spin dynamics, exciton fine structure, exchange interaction

S emiconducting heterostructures are at the heart of modern optoelectronic devices. ${ }^{1,2}$ Recently, it has become possible to prepare heterostructures from atomically thin two-dimensional (2D) materials. Solutionbased routes to such materials, in the form of colloidal
Received: April 27, 2019

Accepted: September 6, 2019

Published: September 6, 2019 
a

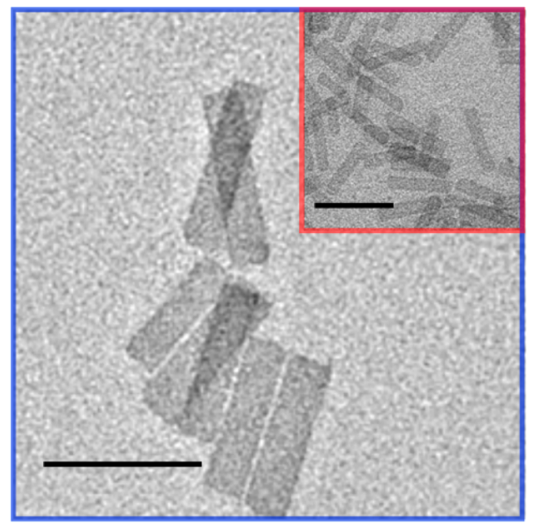

b

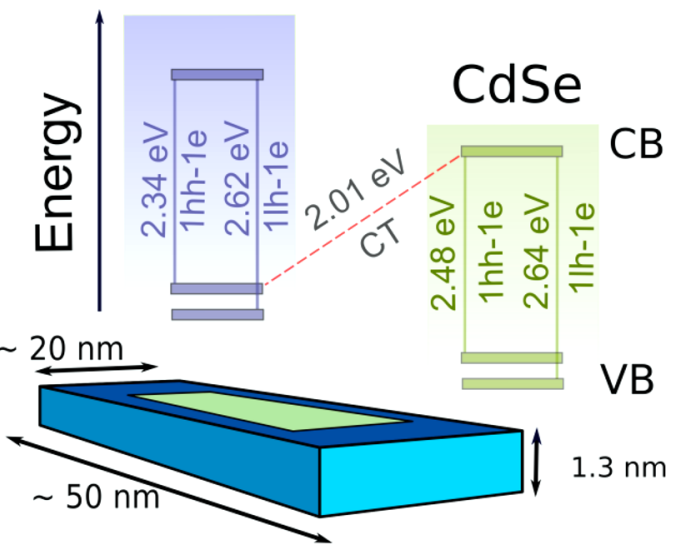

C

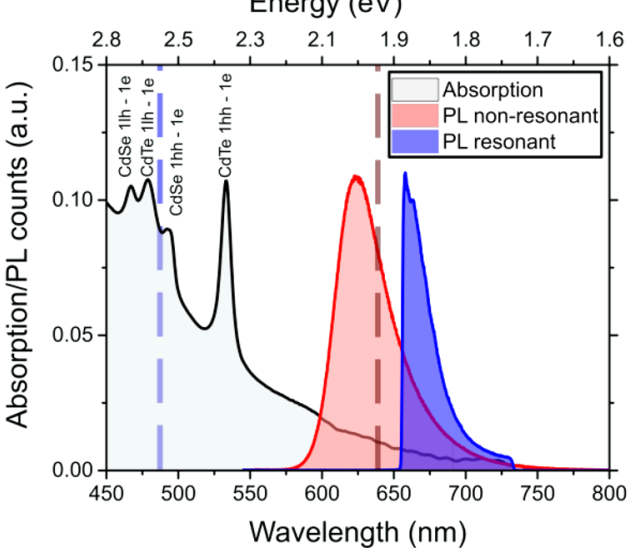

d

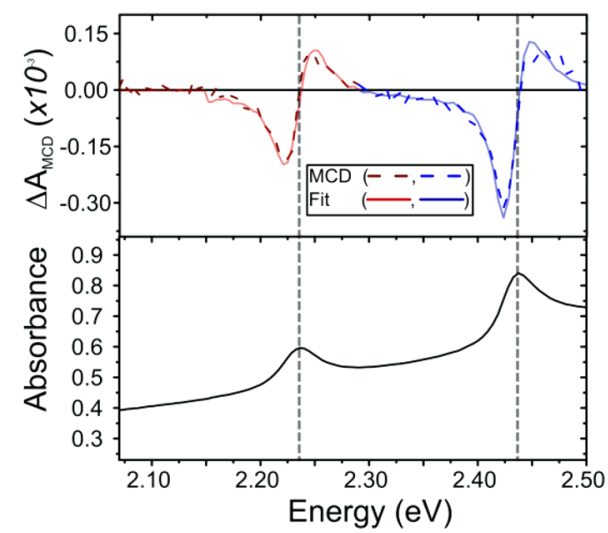

Figure 1. Optical and structural characterization of CdSe/CdTe four-monolayer nanoplatelets (NPLs). (a) Transmission electron microscopy image of core-crown (NPLs) of $\sim 50 \times 20 \mathrm{~nm}$ in size. Inset (red border) shows the CdSe NPL core which is $\sim 35 \times 12 \mathrm{~nm}$ in size. Scale bar in both images is $50 \mathrm{~nm}$. (b) Cartoon depicting excitonic transitions of CdSe/CdTe core-crown NPLs and their respective energies at $4 \mathrm{~K}$. Based on the energetic offsets, electrons are expected to lie in the CdSe conduction band with holes in the CdTe crown. Recombination occurs across the type-II or quasi-type-II heterojunction. (c) Absorption and PL spectra of core-crown NPLs at cryogenic temperatures ( 4 and $1.6 \mathrm{~K}$, respectively). The absorption spectrum (black curve) shows four peaks arising from the CdSe, CdTe heavy/light hole-electron transitions as marked, along with a broad tail out to $750 \mathrm{~nm}$ arising from IX state absorption. The dashed lines indicate the excitation wavelengths used for off-resonant $(480 \mathrm{~nm})$ and quasi-resonant $(640 \mathrm{~nm})$ excitation. The PL spectrum (blue curve) at $1.6 \mathrm{~K}$ shows a slight asymmetry, and the shape of the spectrum in the case of resonant excitation is obscured by the presence of a filter to cut excitation light. (d) Magneto circular dichroism spectrum (MCD; dashed line) of $4 \mathrm{ML}$ CdSe/CdTe NPLs dispersed in hexane. Measurements are performed at room temperature with a magnetic field strength of $1.6 \mathrm{~T}$. The measured MCD is fit using the derivative of the absorption peaks at $2.24 \mathrm{eV}$ (red) and $2.42 \mathrm{eV}$ (blue), which are well matched with the center of the absorption bands of the pure CdSe and CdTe domains, respectively. From this, the solution and orientation-averaged Landé $g$-factors of the individual core and crown can be estimated to be 0.35 and 0.22 , respectively; this is found to be independent of the applied field (SI, S5).

nanoplatelets (NPLs), have garnered significant interest due to their relative ease of fabrication and tunable optical properties via atomic thickness and composition control. ${ }^{3,4}$ Much of the effort has been directed toward cadmium chalcogenide heterostructures, typically consisting of a $\mathrm{CdX}(\mathrm{X}=\mathrm{S}$, Se, $\mathrm{Te}$ ) core surrounded by an alternate $\mathrm{CdX}$ material. The second material is typically grown either in the strong confinement direction on the exposed face of the NPL (termed core-shell) or laterally in plane around the core where the confinement is weaker (termed core-crown). ${ }^{5}$ Although the exciton-fine structure and spin physics of CdSe NPLs have been explored, ${ }^{6,7}$ much less remains known about core-crown NPLs. Of particular interest are $\mathrm{CdSe} / \mathrm{CdTe}$ core-crown NPLs, where as a result of the respective energetic offsets the highest valence band states (holes) lie in the CdTe crown and the lowest conduction band states (electrons) lie in the CdSe core, representing a type-II (or quasi-type-II) band alignment. ${ }^{8,9}$ These materials can support excitons built from the spatially separated charge carriers and are termed indirect (IX) or charge transfer (CT) excitons (the former terminology will be used throughout the paper). These hetero-NPLs, whose thickness can be tuned between three and five monolayers, have room-temperature quantum yields (PLQY) in excess of $70 \%$, long exciton radiative lifetimes ( $<200 \mathrm{~ns})$, and extremely low lasing thresholds $\left(<50 \mu \mathrm{J} / \mathrm{cm}^{2}\right) .{ }^{10-15}$ Consequently, they have been proposed as ideal candidates for photocatalysts, luminescent solar concentrators, and light-emitting diodes as well as being a model system for investigation of excitons in $2 \mathrm{D}$ heterostructures. ${ }^{16-19}$ Furthermore, because one carrier can be shared between two excitons, type-II nanostructures are promising materials for quantum gates operating through the controlled interaction of pairs of qubits encoded on 
neighboring excitons. ${ }^{20-22}$ However, in order to assess the potential of type-II NPLs for optoelectronic or quantum devices, the magneto-optical properties of the lowest energy band edge IXs must be understood.

Here, using a combination of time- and polarization-resolved spectroscopy at high magnetic fields $(30 \mathrm{~T})$ and cryogenic pump-probe spectroscopy, we address the IX fine structure (EFS) and the spin physics of $\mathrm{CdSe} / \mathrm{CdTe}$ NPLs. Resonant excitation of the lowest energy $\mathrm{CdSe} / \mathrm{CdTe} \mathrm{IX}$ transition reveals that, at zero-field, the EFS of the bright IX consists of two linearly polarized bright states, energetically split by $\sim 50$ $\mu \mathrm{eV}$ due to an anisotropic exchange interaction (AEI). Upon application of low magnetic fields $(<5 \mathrm{~T})$, an interplay between the AEI and Zeeman interaction results in an unusual coupling of excitons to circularly polarized light of opposite helicity with respect to that used to excite. At higher magnetic fields (5-30 $\mathrm{T})$, Zeeman interactions dominate over AEI and any spin mixing. The symmetry of IX eigenstates evolves with the magnetic field from linear symmetry at zero field to circular symmetry at high Zeeman splitting. From this regime, we determine the IX Landé factor, $g_{\text {ex }} \approx 0.7$. Complementary modeling of the NPLs magneto-optical response allows determination of the IX spin lifetime and EFS splitting, corroborating its amplitude being in the tens of $\mu \mathrm{eV}$ range. In addition, measurement of the spin relaxation rate as a function of magnetic field reveals an unusual $B^{-3}$ dependence. The origin of this cannot be assigned but potentially results from coupling between the exciton spin and acoustic phonons. Finally, the photoluminescence (PL) is found to weakly depend on the magnetic field, suggesting the small influence of intra-EFS relaxation toward optically inactive excitons, even at large field strengths.

Our results provide a direct picture of IX fine structure and spin dynamics at the interface of a $2 \mathrm{D}$ lateral heterostructure. The uncovering of a small EFS splitting coupled with high PLQY and simple batch sample fabrication may allow for heteroNPLs to be used in devices for quantum bit manipulation or for the production of entangled photons. ${ }^{23-25}$ With careful growth control and heterostructure design, regimes of quantum emission can indeed be reached in such systems. ${ }^{26}$ More generally, the discovery of linearly polarized exciton states makes these materials particularly appealing for optoelectronic applications such as liquid crystal displays and biological visualization agents. ${ }^{27,28}$ Typically, spherical (or nearly spherical) colloidal quantum dots emit circularly polarized light, and hence, filters with their associated losses must be used to analyze the linearly polarized emission. ${ }^{29,30}$ In addition, the work provides a framework for investigating the low temperature physics and spin dynamics of NPLs and more generally $2 \mathrm{D}$ heterostructures especially when the emission is broad and beyond study with conventional techniques.

\section{RESULTS AND DISCUSSION}

NPL Structural and Optical Characterization. Figure 1a shows a transmission electron microscopy (TEM) image of CdSe/CdTe NPLs (CdSe NPL core inset) synthesized using a method detailed previously. The NPLs consist of a $\sim 35 \times 12$ $\mathrm{nm} \mathrm{CdSe}$ core on whose edges a CdTe crown is laterally grown; this results in a platelet with total dimension of $\sim 50 \times$ $20 \mathrm{~nm}$ (see schematic representation in Figure 1b). The NPLs crystallize in a zinc blende structure with CdTe crown grown on the facets of the CdSe core that are perpendicular to the [001] direction. ${ }^{15}$ All platelets considered in this study have a thickness of four monolayers (4 MLs). In order to minimize aggregation, NPLs were mixed with a $\sim 20 \%(\mathrm{w} / \mathrm{v})$ polystyrene solution before being depositing as dilute films via either blade or spin coating. The dispersion of platelets within the film is confirmed via transmission electron microscopy and PL lifetime measurements which show no change in the PL lifetime between solution and film (see Figure S3). Figure 1c shows the absorption spectrum of a thin film of core-crown NPLs at $4 \mathrm{~K}$. Four prominent transitions, illustrated in Figure 1c, can be observed at $\sim 530 \mathrm{~nm}(2.34 \mathrm{eV})$ and $\sim 473 \mathrm{~nm}(2.62$ $\mathrm{eV}$ ) resulting from the $\mathrm{CdTe}$ crown electron-heavy hole (e$\mathrm{hh})$, electron-light hole (e-lh) transitions, and at $\sim 500 \mathrm{~nm}$ $(2.48 \mathrm{eV})$ and $\sim 470 \mathrm{~nm}(2.64 \mathrm{eV})$ resulting from the e-hh and e-lh transition in the CdSe core. In addition, there is a broad tail of transitions extending to $\sim 750 \mathrm{~nm}$. As shown in previous studies this does not arise from light scattering but from absorption of the IX state. ${ }^{31}$ Measuring the absorption of left and right circularly polarized light under a weak magnetic field (magneto-circular dichroism spectroscopy, MCD) ${ }^{32,33}$ also allows us to determine the Landé $g$-factor, $g_{\mathrm{ex}}$, of e-hh excitons in the individual $\mathrm{CdSe} / \mathrm{CdTe}$ domains. This is shown for heteroNPLs in solution at room temperature in Figure 1d where a characteristic "first derivative" of absorption feature at room temperature (dashed line) arises in the MCD spectrum similar to that observed in $0 \mathrm{D}$ dots. ${ }^{34}$ Fitting the first derivative (dashed line) of the absorption band for the lowest energy $\mathrm{CdSe}$ (blue) and CdTe (red) domain exciton transitions as a function of magnetic field gives $g_{\text {ex }}$ of 0.35 and 0.22, respectively (see SI, S5, for further details).

In contrast to $\mathrm{CdSe}$ core or $\mathrm{CdSe} / \mathrm{CdS}$ core-shell NPLs, the PL of core-crown CdSe/CdTe NPLs is broad and significantly red-shifted from the absorption. The red- and blue-shaded spectra show the emission of NPLs for excitations above the band gap at $480 \mathrm{~nm}$, and in quasi-resonance with the IX absorption at $640 \mathrm{~nm}$ (at $1.6 \mathrm{~K}$ ). In the case of quasiresonant excitation the presence of a filter to cut laser excitation limits absolute determination of the PL shape. We remark that on cooling to $1.6 \mathrm{~K}$ the entire $\mathrm{PL}$ spectrum narrows (fwhm $\sim 130 \mathrm{meV}$ at room temperature to $\sim 65 \mathrm{meV}$ at $1.6 \mathrm{~K})$, but even at these low temperatures it is still relatively broad and featureless. In comparison, the line width of direct excitons measured in diluted phases of chalcogenide nanocrystals (NCs) is typically in the few meV range. ${ }^{35,36}$ This limits investigation of the fine structure via conventional techniques such as spectral shifting, narrowing, and splitting of the PL peak at low temperature and on the application of weak (or moderate) magnetic fields. ${ }^{6,30,37,38}$ Consequently, we chose to investigate the EFS, primarily via magneto-optical experiments exploiting the effect of the magnetic field on the polarization of PL emission under both off-resonant and quasiresonant excitation. We note that for quasi-resonant excitation of the IX transition there is no charge (carrier) transfer accompanying the IX generation process and momentum relaxation pathways are strongly limited, making possible the preparation of IXs in well-defined spin configurations.

Magneto-Optical Effects: Experimental Results and Modeling. The degrees of circular and linear polarization (DCP and DLP, respectively) are useful quantitative tools for characterizing the EFS of semiconductor materials and are central to this work; ${ }^{39-42}$ we define them in the usual convention as DCP $=\left(I_{\sigma+}-I_{\sigma-}\right) /\left(I_{\sigma+}+I_{\sigma-}\right)$, whatever the circular polarization of the excitation, where $I_{\sigma+}\left(I_{\sigma-}\right)$ is the intensity of $\sigma^{+}\left(\sigma^{-}\right)$circularly polarized component of the 
emission and DLP $=\left(I_{/ /}-I_{\perp}\right) /\left(I_{/ /}+I_{\perp}\right)$, with $I_{/ /}$and $I_{\perp}$ denoting the intensity of the emission analyzed in a direction parallel or perpendicular to the linearly polarized excitation. In nanoparticles, the application of a strong magnetic field induces a Zeeman splitting in the exciton states which manifests itself as a contrast between $\sigma^{+}$and $\sigma^{-}$emission, under $\sigma^{+}\left(\sigma^{-}\right)$excitation. ${ }^{42}$ Figure $2 \mathrm{a}$ shows $\sigma^{+}$and $\sigma^{-}$PL
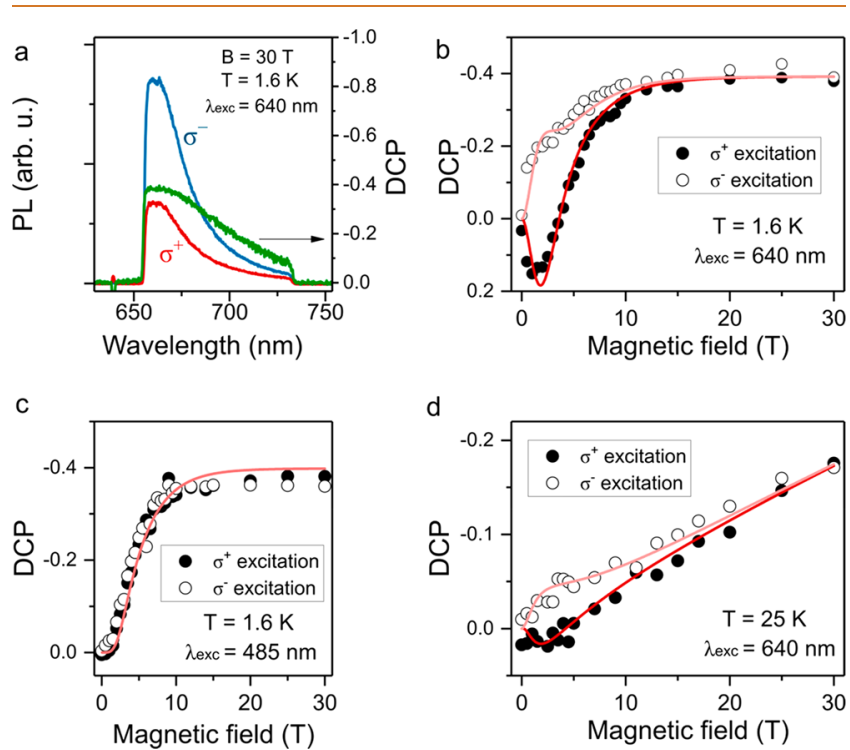

Figure 2. Magneto-optical responses of CdSe/CdTe NPLs dispersed in polystyrene films. (a) Spectrally resolved $\sigma^{+}$and $\sigma^{-}$ emissions at $1.6 \mathrm{~K}$ and $30 \mathrm{~T}$ and the associated degree of circular polarization, DCP, as a function of wavelength (green line); see main text for definition of DCP. (b, c) DCP as a function of magnetic field strength under quasi-resonant excitation $\left(\lambda_{\text {exc }} \approx 640\right.$ $\mathrm{nm}, \mathrm{cw}$ ) at 1.6 and $25 \mathrm{~K}$ respectively. (d) Measured $\mathrm{DCP}(\mathrm{B})$ under nonresonant excitation $\left(\lambda_{\text {exc }} \approx 485 \mathrm{~nm}, \mathrm{cw}\right)$. In $(\mathrm{b})-(\mathrm{d})$, the red and light red solid lines are fits arising from EFS spin relaxation modeling (see main text and Supporting Information for model description). All DCPs are determined at $660 \mathrm{~nm}$.

spectra of core-crown CdSe/CdTe NPLs under resonant $\sigma^{+}$ excitation at $1.6 \mathrm{~K}$ and $30 \mathrm{~T}$ as well as the obtained DCP spectrum. Across the whole emission band, the DCP diminishes from $40 \%$ at the peak $(\sim 660 \mathrm{~nm})$ to $10 \%$ at the low energy tail. However, in this work we shall limit the magneto-optical study and analysis to emission in the interval $660-670 \mathrm{~nm}$ where the DCP only slightly varies $(<1 \%)$. In this region, we can tentatively assign the emission band to be from a single species, that is, a neutral exciton whose transition is inhomogeneously broadened. The strong quenching of DCP at longer wavelengths may arise from the overlap of emission between several different PL bands, the origin of which is beyond the scope of this study.

The dependence of the DCP on the applied magnetic field along the propagation direction of the excitation light provides several insights into the EFS: first, at $1.6 \mathrm{~K}$, the evolution of the DCP under $\sigma^{+}$quasi-resonant excitation (Figure $2 \mathrm{~b}$ ) is non monotonic and shows a pronounced positive peak at $B \sim 2 \mathrm{~T}$, whereas the $\mathrm{DCP}(\mathrm{B})$ observed for a $\sigma^{-}$excitation continuously increases. At high field, the DCPs from $\sigma^{-}$and $\sigma^{+}$excitations both converge toward a limiting value of $\sim 0.39$. On increasing the temperature to $25 \mathrm{~K}$, the DCP is globally weaker than at $1.6 \mathrm{~K}$ and does not reach saturation in the same range of magnetic field (Figure 2d); moreover, the amplitude of the low field peak is strongly reduced. Such effects are absent in the nonresonant scheme (Figure 2c), indicating that under resonant excitation polarization-induced selection is achieved as helicity is changed. This also suggests that at least two excitonic states contribute to the emission, whose thermalization is mainly driven by spin relaxation. As detailed later, we will show that the inversion in the DCP observed at $1.6 \mathrm{~K}$ relates to a competition between the spin relaxation and excitonic recombination dynamics. Finally, we also note that the DCPs measured under nonresonant excitation show the same $B$ dependence for both $\sigma^{+}$and $\sigma^{-}$polarizations. They remain negative and saturate at a value of $\sim 40 \%$, significantly lower than $75 \%$ achievable in assemblies of spherical QDs. ${ }^{42}$

In light of the clear excitation polarization effect under resonant excitation, another key feature of the data in Figure 2 is the observation of a null DCP value at zero field for resonant excitation, pointing to an emission arising from linearly polarized states in absence of magnetic coupling. This loss of circular symmetry in emission also appears in resonant DLP measurements when no field is applied. Polar plots of emission intensity are shown in Figure $3 \mathrm{a}$. The polarization of the exciting beam is set with a polarizer (angle $\beta$ with respect to the laboratory horizontal axis) and the PL analysis is performed, using a rotating half-waveplate and a polarizer whose direction remains fixed along the "best response" axis of the spectrometer (vertical axis). The combination of the waveplate and polarizer acts as a single analyzer. When repeating the experiment for different values of $\beta$, we observe that the maximum of PL intensity is always reached when the analysis is performed along the direction of the exciting light, defined by the angle $\beta$, and a high DLP $(40 \%<\operatorname{DLP}<70 \%)$ is evidenced. Importantly when the excitation is tuned out of resonance the DLP is found to be close to zero (filled circles in Figure 3a) and no preferential polarization appears in the PL.

Bright-exciton states hybridization has been observed in epitaxial and elongated colloidal dots ${ }^{30,38,43-45}$ and although it can have many different specific origins, it is most generally always related to a reduction in the nanostructure symmetry, a point which will be discussed in subsequent sections. Following hybridization the resulting lowest energy bright states are then linear combinations of heavy-hole exciton states characterized by the angular momentum $M_{s}= \pm 1$ (generally expressed as $| \pm 1\rangle)$. To such combinations correspond non degenerate $|X\rangle$ and $|Y\rangle$ states, which are dipole-active along some of the high symmetry crystallographic axes of the NC. In order to first rationalize the DCP behavior observed in corecrown NPLs and extract the relevant EFS parameters, we suggest the following, closely accounting for the general symmetry of the platelets: the NPL EFS forms a system of $|X\rangle$, $|Y\rangle$ states lying in the NPL plane associated with linearly crossed polarized transitions and split in energy by an amount $\delta_{1}$ (fine structure splitting; Figure $3 \mathrm{~b}$ ). In this framework, the general magneto-optics of the NPLs films is already relatively well described by considering ideally organized ensembles of "in-plane" (horizontal) NPLs placed in the magnetic field applied in the direction of propagation of the exciting light and perpendicular to the sample substrate ( $z$ direction). We then suggest that important corrections to the DCP curves might arise from the contribution of the NPLs having different orientations and a different response to the magnetic field.

Let us first consider the case of horizontal NPLs; i.e., all the NPLs are aligned face-on to the substrate. $|X\rangle$ and $|Y\rangle$ states experience a Zeeman interaction; the Hamiltonian describing 


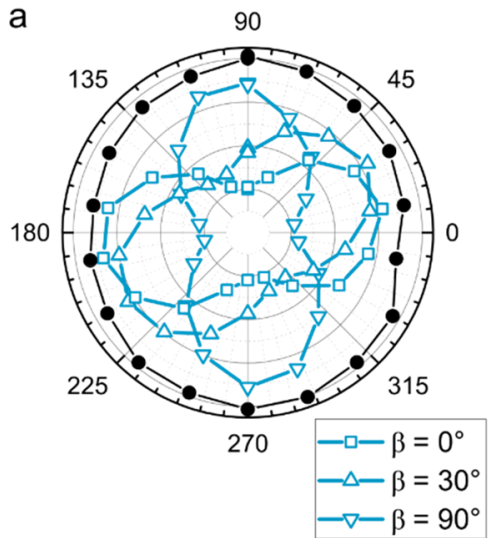

b

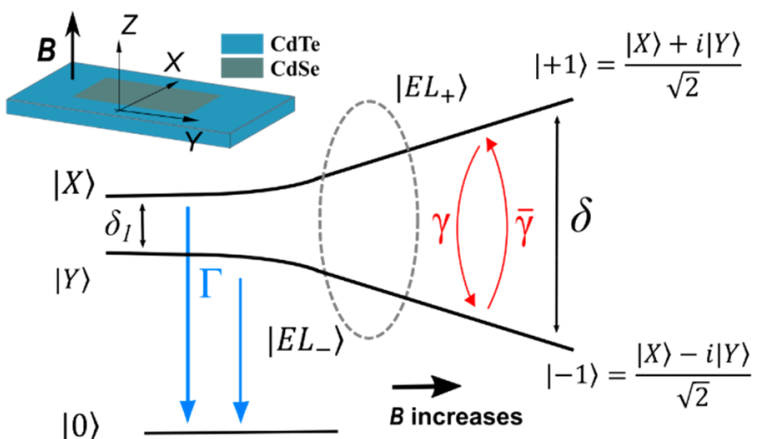

Figure 3. Degree of linear polarization (DLP) at zero field and schematic of the lowest exciton energy sublevels in CdSe/CdTe NPLs as a function of the Zeeman splitting. (a) Polar plot of PL intensities obtained from linearly polarized excitation at zero magnetic field. DLP measurements were reproduced for different orientations of the linear incident polarization, $\beta$ (angle between the laboratory horizontal axis, taken as a reference, and the axis of incident polarization). Open squares and up and down triangles correspond to $\beta$ equal to $0^{\circ}, 30^{\circ}$, and $90^{\circ}$, respectively, for a resonant excitation. The solid circles show the measurements performed for an off-resonant excitation. In this latter case, the response is independent of $\beta$. (b) Schematic of EFS levels and experimental configuration. The magnetic field remains perpendicular to the plane of "flat-lying" NPLs. At $B=0$, the EFS is composed of $|X\rangle$ and $|Y\rangle$ states associated with linearly polarized transitions. Their energies are split by the fine structure splitting, $\delta_{1}$. The dipoles associated with $|X\rangle$ and $|Y\rangle$ are taken aligned with the NPLs edges along the larger dimensions. With increasing magnetic field both states first acquire elliptic symmetry (IEL +$\rangle$ and $|E L-\rangle$ states defined in eqs $2 \mathrm{a}$ and $2 \mathrm{~b}$ ) with principle axes still aligned with $X$ and $Y$ directions (region delimited by the dashed ellipse, with $\delta_{z} \approx \delta_{1}$ ). At higher field $|E L+\rangle$ and $|E L-\rangle$ transform toward $|+1\rangle$ and $|-1\rangle$ owning pure circular symmetry and are accessible using $\sigma^{+}$and $\sigma^{-}$circular light, respectively. The energy separation between split states, $\delta(B)$, is $\delta(B)=\left(\delta_{1}{ }^{2}+\delta_{z}{ }^{2}\right)^{1 / 2}$. From the fit of the DCP curves, one finds, for the exciton longitudinal Lande factor, $g_{z} \approx 0.7$, meaning that $\alpha \approx 40^{\circ}$ for $B \approx 6 \mathrm{~T}$; this provides an order of magnitude of the $B$ field required to generate quasi-circularly polarized transitions in the system.

the exciton states in the presence of the longitudinal field (Faraday geometry) is ${ }^{38}$

$$
\begin{aligned}
\hat{H} & =\left(\hbar \omega_{0}-\frac{\delta_{1}}{2}\right)|X\rangle\left\langle X\left|+\left(\hbar \omega_{0}+\frac{\delta_{1}}{2}\right)\right| Y\right\rangle\langle Y| \\
& +\left(\frac{\delta_{z}}{2}\right)(|+1\rangle\langle+1|-|-1\rangle\langle-1|)
\end{aligned}
$$

where $| \pm 1\rangle$ are the states with $M_{\mathrm{s}}= \pm 1 . \delta_{z}$ is the Zeeman splitting given by $g_{z} \mu_{\mathrm{B}} B_{z}$ with $g_{z}$ being the exciton longitudinal $g$-factor. The new eigenstates, denoted $|E L+\rangle$ and $|E L-\rangle$, are then: ${ }^{46}$

$$
\begin{aligned}
& |E L+\rangle=\sin \alpha|X\rangle+i \cos \alpha|Y\rangle \\
& |E L-\rangle=\cos \alpha|X\rangle-i \sin \alpha|Y\rangle
\end{aligned}
$$

and have respective energies $E_{ \pm}=\hbar \omega_{0} \pm \frac{1}{2} \sqrt{\delta_{1}^{2}+\delta_{z}^{2}}$. The field dependent angle, $\alpha$, is defined as $\frac{1}{2} \arctan \left(\frac{\delta_{z}}{\delta_{1}}\right)$. With increasing field, the $|X\rangle$ and $|Y\rangle$ states acquire elliptic symmetry, explaining the increase in the DCP. At high field $\delta_{z} \gg \delta_{1}$ so $\alpha \rightarrow \pi / 4$, the circular symmetry of the states is restored and the DCP reaches its maximum. The relaxation between the higher and lower lying energy states is governed by the rates $\gamma$ and $\bar{\gamma}$ (see Figure $3 \mathrm{~b}$ ) where $\bar{\gamma}=\gamma e^{-\delta(B) / k_{\mathrm{B}} T}$; the factor $\delta(B)=\left(\delta_{1}{ }^{2}+\delta_{z}{ }^{2}\right)^{1 / 2}$ is required by the Boltzmann statistics for phonons of the bath at temperature $T$. In this picture, the rate that governs the dynamics of the DCP is the average relaxation rate, $(\gamma+\bar{\gamma}) / 2$ (see model in SI, S9), that identifies with the spin relaxation decay rate, $\Gamma_{\mathrm{S}}=1 / \tau_{\mathrm{S}}\left(\tau_{\mathrm{S}}\right.$ is the exciton spin relaxation time). The relaxation mechanism corresponds to a process where the electron and the hole flip their spin simultaneously by absorbing or emitting an acoustic phonon ("direct" spin flip). This is thought to be the most likely process in nanocrystals where (i) the processes relying on the exciton motion (as for quantum-well excitons) are suppressed $^{47}$ and (ii) the relativistic effects that lead to the spin-flip of single carriers, through spin-orbit coupling, has been shown to be inefficient. ${ }^{48}$ For these reasons, we will restrict our analysis to the dynamics between bright-states and neglect processes that may act on single carriers or be responsible for transfers between $|X\rangle$ and $|Y\rangle$ states and their "dark" counterparts. Finally, as a first approximation, we also assumed equal oscillator strength for states, $|\mathrm{EL} \pm\rangle$, associated with the upper, and lower energy bright states branches. We also show, in the SI section (S11), that field renormalization effects that result from the inner-outer dielectric contrast can be considered as negligible.

The DCP(B) evolution shown in Figure $2 b, d$ is unusual but can, based on the above, be rationalized as follows. Once the polarization of the excitation $\left(\sigma^{+}\right.$or $\left.\sigma^{-}\right)$is fixed, the interplay between the exciton recombination and spin relaxation governs the dynamics and occupation of the $|E L+\rangle$ and $|E L-\rangle$ states. The inversion in the DCP sign, responsible for the positive dip observed at low field under $\sigma^{+}$excitation, should hence be associated with a decrease of $\tau_{\mathrm{S}}$ with increasing magnetic field (note that this decrease is confirmed and commented on below). When we consider $\tau_{\mathrm{S}}$ as being fixed, the relative weight of $\sigma^{+} / \sigma^{-}$emission will also strongly depend on the thermal occupation of the EL+ and EL- levels. This latter quantity can be estimated from the ratio between the total energy splitting, $\delta(\mathrm{B})$, and the characteristic thermal width, $k_{\mathrm{B}} T$. Spin relaxation will lead to larger DCPs when $\delta$ is larger than $k_{\mathrm{B}} T$. Based on this, we expect lower DCP values at higher temperatures (for a given $B$ value), and in addition, the average difference between the DCP values obtained using left and right-hand polarized excitation should decrease (see Figure $2 b, c$ ). At high magnetic 
fields, and for $T=1.6 \mathrm{~K}$, the observation of equal DCP for $\sigma^{+}$ and $\sigma^{-}$excitation indicates that the spin relaxation becomes efficient enough to equilibrate the $|E L+\rangle$ and $|E L-\rangle$ state populations within a time shorter than the exciton lifetime, $\tau_{\mathrm{R}}=1 / \Gamma$. At $B=30 \mathrm{~T}, k_{\mathrm{B}} T \ll \delta(\mathrm{B})$ meaning that, very rapidly, the lowest state is populated with the majority of the PL intensity coming from this state. Under those conditions, a DCP value of 1 is expected. The fact that the experimentally obtained value differs from 1 (DCP $\approx 0.39$ ) likely finds its origin in the existence of NPLs that do not lie horizontally. Finally, in the nonresonant scheme the populations of $|\mathrm{EL}+\rangle$ and $|E L-\rangle$ states no longer depend on the incident polarization and the $\sigma^{+} / \sigma^{-} \operatorname{DCP}(\mathrm{B})$ curves are identical (Figure 2c). This is likely due to a complex cascade of spin relaxations following excitation into the domain exciton states. We remark that, as $\tau_{S} \rightarrow 0$, the $|E L+\rangle$ and $|E L-\rangle$ populations immediately reach thermal equilibrium whatever the incident beam polarization and the situation of nonresonant excitation is recovered (see Figure S12).

Complete consideration of the effects of orientational disorder is beyond the scope of the present work. However, to gain an improved agreement between simulated DCPs and the experimental data, refinements to model presented above can be considered, namely via the inclusion of a second population made of NPLs with an "edge" configuration that lies in a vertical plane. In this case, we consider the respective fractions of "in-plane" and "edge" NPLs that compose the medium as $n_{\text {hor }}$ and $n_{\text {ver }}=1-n_{\text {hor }}$, respectively. These "edge" NPLs deserve a particular attention with respect to the magnetic coupling. The in-plane magnetic field causes a mixing of bright ( $|X\rangle,|Y\rangle$ states) and dark excitons $\left(\left|X^{\prime}\right\rangle,\left|Y^{\prime}\right\rangle\right.$ states), that are formed, for their part, from the hybridization of $M_{s}=$ \pm 2 states. The resulting wave function is, in its general form, a linear combination of bright and dark states but exhibit a structure where the $|X\rangle$ (or $|Y\rangle$ ) part is preserved as a whole whatever the magnetic field amplitude ${ }^{38}$ (SI, S9). The coupling to light operates through either the $|X\rangle$ or the $|Y\rangle$ component in the wave function so that an edge NPL keeps absorbing or emitting light along the $X$ or $Y$ direction as the amplitude of $B$ is changed. Hence, the vertical population will tend to lead to a global reduction in the measured DCP preventing the obtainment of DCP = 1 in the high field limit.

Fits of the DCP curves obtained within the above framework are also shown in Figure $2 b-d$ (see SI, S9, for the derivation of the DCP expressions as well as for the description of the adjustment procedure). The following set of EFS parameters is found to simultaneously best fit all curves: $\delta_{1} \approx 60 \mu \mathrm{eV}, \tau_{\mathrm{R}} \approx$ $620 \mathrm{~ns}, g_{z} \approx 0.7$. We also find $n_{\text {hor }} \approx 0.24$, suggesting a higher amount of NPLs with a vertical orientation within the polystyrene matrix. This is further supported by transmission electron microscopy (TEM) images of the films obtained following ultramicrotomy (see Figure S4) where it is also seen that edge- and flat-lying NPLs are the majority population. Varying the NPLs density inside the film could also allow us to change the $n_{\text {hor }} / n_{\text {ver }}$ ratio, which would directly affect the high field DCP value. ${ }^{7}$ Measurements as a function of the NPLs concentration were not attempted in the present work. At 1.6 $\mathrm{K}, \tau_{\mathrm{R}}$ is found to be on the same order of magnitude as the average lifetime extracted from the multiexponential fit of the emission $\bar{\tau}_{\mathrm{R}}=\left(\sum_{i} N_{i} \tau_{i}\right) /\left(\sum_{i} N_{i}\right) \approx 340 \mathrm{~ns}\left(A_{i}\right.$ and $\tau_{i}$ are the amplitudes and decay times of each component and $N_{i}=A_{i} \tau_{i}$ is the number of photons emitted in the channel $i$ ). The model also predicts a DLP $=0.5$ at $B=0$ which does not depend on the ratio $n_{\mathrm{hor}} / n_{\mathrm{ver}}$. This is generally in good agreement with the values extracted from Figure $3 \mathrm{a}$, although we find there to be a degree of variation in the experimental data that, on average, indicates this value to be slightly lower. The DLP dependence on the magnetic field was also measured (Figure S13) and it is consistently found that the DLP decreases as the magnetic field is increased and states gain in circular symmetry. However, the DLP value at high field does not reach zero, as would be expected for ensembles of flat and edge-lying NPLs with random orientations around the $z$ axis, instead saturating at $\sim 15-20 \%$. The existence of this residual DLP at high field can be readily explained by taking into account a peaked distribution in the orientation function (see simulation in SI, S9). Local order may then also explain DLPs greater than 50\% at zero field that should be observed for given positions in the sample; it is important to note that an in-plane global orientation of NPLs has no influence on the DCP properties, which were indeed found to be identical across the sample (SI, S9).

Surprisingly, the bright-state splitting, $\delta_{1}$, is found to be relatively large despite the spatial separation of the electron and the hole and the associated reduced overlap of their wave functions. This value compares more typically with the fine structure state splitting found in epitaxially grown $\mathrm{Ga}(\mathrm{In}) \mathrm{As}$ type-I quantum dots $\left(\delta_{1} \approx 0.1 \mathrm{meV}\right)^{5,38,44,49,30}$ but remains 1 order of magnitude lower than typical splittings measured in highly confined CdSe NCs. ${ }^{51}$ The presence of a high dielectric contrast between the NPL and the outside medium is expected to lead to a magnification of all Coulomb interactions. ${ }^{52-54}$ For instance, for direct Coulomb integrals, simulations suggest values over half those in type-I NPLs but still comparable to type-I spherical NCs, ${ }^{5}$ although these calculations do not take into account the exchange interaction term responsible for the lifting of degeneracy between $| \pm 1\rangle$ states. To the best of our knowledge most studies on type-II NCs have so far focused on the energetics of the excitonic transitions, associated binding properties, or exciton-exciton interactions. ${ }^{55-59}$ One recent work has addressed the EFS of type-II NCs having ellipsoidal symmetry (GaAsSb-capped InAs quantum dots) with an aspect ratio similar to the ones characterizing $\mathrm{CdSe} / \mathrm{CdTe}$ NPLs. Here it was shown that in the absence of dielectric confinement, the long-range contribution of the exchange interaction could already be responsible for splittings of tens of $\mu \mathrm{eV} .^{60}$

Returning to the emission properties, we also find that the PL decay is best described by a triexponential decay (across all field $B=0-30 \mathrm{~T}$ ). The multicomponent decay is not specific to type-II NPLs; similar behavior is observed, at zero field, in Cd-based homo- and heteroplatelets supporting direct excitons. $^{4,35}$ In core-shell CdS/CdSe NPLs, the strong multiexponential decays were attributed to charge carrier separation and release, responsible for long time scales delayed emission. ${ }^{61}$ However, the decay's structure was found to be much more complex and was best fitted using a power law. We now first comment on the PL dependence on the magnetic field before addressing the effect of temperature. In the studied $\mathrm{CdSe} / \mathrm{CdTe}$ NPLs, the total PL intensity is found to remain constant under resonant excitation within the explored $B$ range. The PL lifetimes are very weakly affected by the magnetic field, as seen in Figure 4. At $1.6 \mathrm{~K}$ the decay constants do not vary (within experimental error) and the main change is an $\sim 20 \%$ variation of the amplitude associated 

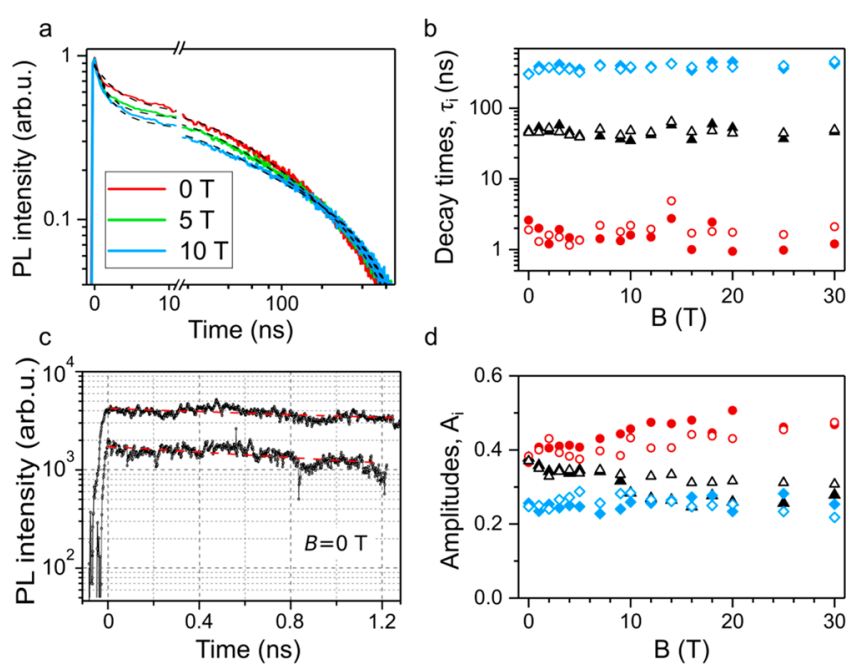

Figure 4. Photoluminescence (PL) dynamics as a function of the magnetic field $(T=1.6 \mathrm{~K})$. The excitation is tuned to $640 \mathrm{~nm}$ (quasi-resonant scheme). (a) Decay of the total PL intensity shows little variation with increasing magnetic field. The experimental curves are best-fitted using three exponentials $\left(A_{i}, \tau_{i}, i=1-3\right)$. (b) Decay kinetics: evolution of the decay constants, $\tau_{\mathfrak{j}}$, with the magnetic field, $B$. (c) PL decay obtained under quasi-resonant excitation $\left(\lambda_{\mathrm{ex}}=610 \mathrm{~nm}, \sigma^{+}\right.$excitation $)$of the IX transition at $T=$ $1.6 \mathrm{~K}$ and for $B=0$. The curves correspond to two different probed regions. The dash lines are monoexponential fits with a decay constant, $\tau \approx 2.7 \mathrm{~ns}$, in agreement with the measurements performed on a longer time range. (d) Amplitude of components $A_{\mathrm{i}}$ as a function of $B$. In (b) and (d), open/filled symbols are for $\sigma^{+} / \sigma^{-}$excitations, respectively.

with the fastest component, occurring between 0 and $8 \mathrm{~T}$. A similar $B$ dependence has been observed in type-II 0D quantum dots. ${ }^{62,63}$ In NCs, the weak field-dependence of PL dynamics is generally attributed to species such as charged excitons having only bright states which are insensitive to bright-dark state coupling or mixing through the application of the magnetic field. ${ }^{64}$ For charged excitons (trions), one carrier interacts with the other that has two possible spin orientations and the exchange energy splitting at zero field vanishes, leading to states of circular symmetry. ${ }^{38}$ Therefore, in light of the $\mathrm{DCP} / \mathrm{DLP}$ values measured at $B=0$ along with the narrow PL band investigated (with uniform DCP), we deem trions to be a minority among the emitting species at the explored PL energies. A description of the full dynamics of the system and the effect of trions is beyond the scope of the present investigation, but note their presence cannot be fully ruled out. The strong presence of edge lying NPLs is clearly evidenced in STEM images of slices extracted from the studied films using ultramicrotomy (SI, S4). Such NPLs should thus experience a mixing between their optically active states and dark states that is expected to noticeably impact the PL dynamics. As the PL shows very weak variations, we conclude that the magnetooptical coupling remains inefficient; in other words, the magnetic term $g_{z} \mu_{\mathrm{B}} B$ stays below the bright state-dark state splitting, $\Delta$, and placing the $\Delta$ value above the $\mathrm{meV}$. Regardless, the unvarying average PL lifetime with $B$ strongly matches the hypothesis of $\tau_{\mathrm{R}}$ being set as a constant, as was done in the above analysis. As discussed, in this picture, the ratio $\tau_{\mathrm{R}} / \tau_{\mathrm{S}}(B)$ drives the DCP evolution via $\tau_{\mathrm{s}}(B)$ (see below).

More surprising is the absence of $\mathrm{PL}$ variations with temperature. When passing from 1.6 to $25 \mathrm{~K}$, we note small changes that mostly concern the relative amplitudes of the components in the dynamics, not the decay constants themselves (see Figure S9). Importantly, at low field, the long-lived components are not affected when the temperature is raised, and there is no acceleration of the dynamics that would be characteristic of an activated back transfer from a low-lying energy dark level. ${ }^{62,65}$ Our results thus support two possible explanations: a small $\Delta$ value and a relatively strong coupling between the bright and dark states on the one hand or a rather low coupling between each manifold on the other; following the discussion below, this is this latter hypothesis that is retained. In the former case, $\Delta$ has to keep lower than $k_{\mathrm{B}} T$ whatever the temperature. This would first imply $\Delta$ to be vanishingly small $(\ll \sim 140 \mu \mathrm{eV})$ in a system that despite its type-II character has strong bright states splitting. This is in contradiction with the results of magneto-PL indicating much larger $\Delta$. Furthermore, if single electron and hole spin flip were both as fast as in type-I NPLs (characteristic times below 100 ps were evidenced), 6 they should also manifest themselves in the total PL decay at low temperature as a fast component. For large $\Delta\left(\Delta>k_{\mathrm{B}} T\right)$, the initial loss of population would be nearly complete and it should reach $\sim 50 \%$ for quasidegenerate states $\left(\Delta \ll k_{\mathrm{B}} T\right)$. This ultrashort depletion of the bright states is not observed under quasi-resonant excitation of the IX transition (see Figure 4c). Let us remark that the fastest PL component has a dynamic around $\sim 2 \mathrm{~ns}$ that cannot be associated with the individual spin carrier relaxation either for it would be perfectly detectable in the DCP time decay. We thus come to the conclusion that the coupling between bright and dark states has to be inefficient and that the exchange interaction is the main source for exciton spin relaxation. The picture is also strongly supported by the measurement of PL yield as high as $86 \%$ at $4 \mathrm{~K}^{31}$

As an explanation, we suggest that the investigated NPLs share more properties with type-II quantum dots or at least we believe they cannot be directly compared to homonanoplatelets. The dielectric confinement can have a strong impact on the localization of the electronic density close to the interface: the exciton is less mobile and the size of the CdTe region is a parameter of poor influence since there is limited dilution of the charge density in the crown. ${ }^{66}$ As mentioned previously, we expect consequently that the processes relying on the exciton motion, like in QWs, are quasi-suppressed; then the individual electron and hole spin flip processes that are based on the spin-orbit coupling are inefficient like in QDs. ${ }^{48}$ In addition the dielectric contrast favors all Coulomb interactions and, as a consequence, increases exchange integrals. Although further calculations are required to quantify this electrostatic effect we note that the IX binding energy that is a probe of direct Coulomb interaction, should already be large enough to surpass $k_{\mathrm{B}} T \sim 25 \mathrm{meV}$ because the IX PL is visible at room temperature. In highly confined type-II $\mathrm{CdTe} / \mathrm{CdSe} / \mathrm{CdTe}$ nanorods, calculations place the IX binding energy around $\sim 150-200 \mathrm{meV}$ (including the mismatch in $\varepsilon_{\mathrm{r}}$ between the semiconductor and air). ${ }^{59}$ The enhancement effect will be weaker in our samples since polystyrene has a higher dielectric constant, however the exchange interaction can be increased significantly, which should additionally promote the direct spin-flip process (to the best of our knowledge, numerical estimations of exchange integrals are not available in the literature). The dependence of the exciton spin relaxation time on the magnetic field, as a power law, comes as another evidence of the predominance of exchange interaction driven 
processes (see below). Finally, we note that the "freezing" of individual carrier spin flip was also noticed recently in high quality coupled QWs supporting IXs. ${ }^{67,68}$ We also remark that the absence of accepting phonon modes that are able to bridge the gap between the Zeeman states (bottleneck effect) may play a role and hamper intraband relaxation. ${ }^{69}$

Quantum Beat Spectroscopy. Time-resolved optical techniques are very useful in probing the EFS of nanostructures. Here quasi-resonant pump-probe spectroscopy is used as a complementary tool to investigate IX states at zero field. In particular, the coherent superposition of the $|X\rangle$ and $|Y\rangle$ sublevels can be induced by a linearly polarized pump pulse, resonant with the IX transition. The coherent superposition formed is expected to evolve in time with quantum interference effects that manifest themselves as quantum beats in the field of a low intensity probe pulse. ${ }^{70-73}$ Note that similar beats can also be detected in experiments that address the PL decay. ${ }^{74,75}$ For the quasi-resonant scheme and identical linear polarizations of both pump and probe beams, the expression of the differential transmittance takes the form (see SI, S10, for full mathematical derivation)

$$
\begin{aligned}
\frac{\Delta T}{T} \propto & \left(3+\cos ^{2} 2 \theta\right) e^{-\Delta t / \tau_{R}} \\
& +|\sin 2 \theta| \sin 2 \theta \cos \left(\omega_{1} \Delta t\right) e^{-\Delta t / T_{2}}
\end{aligned}
$$

where $\Delta T / T$ is the normalized differential change in transmission $(T)$ with pump on and off, $\Delta t$ is the pumpprobe delay time, $\theta$ is the angle between the incident electric fields and the NPL $X$ axis $(-\pi / 2 \leq \theta<\pi / 2)$, and $\omega_{1}$ is related to the splitting energy, $\delta_{1}$, according to $\hbar \omega_{1}=\delta_{1}$. One should note that eq 3 holds only for a single platelet that lies in the plane of the substrate and is illuminated by pump and probe beams under normal incidence ( $z$ direction). Equation 3 contains two terms whose amplitudes depend on $\theta$ : the first term is a decreasing exponential with a characteristic time corresponding to the IX lifetime and the second one is a damped oscillatory term. The period of the beats (in the second term) is a direct measure of $\delta_{1}$ whereas the beats damping rate is the inverse of the average time, $T_{2}$, during which the superposition is maintained; $T_{2}$ characterizes the dynamics of decoherence between the $|X\rangle$ and $|Y\rangle$ sublevels. In Figure 5 the pump is tuned to $\sim 618 \mathrm{~nm}$, and $\Delta T / T$ is recorded as a function of the pump-probe delay, to $622 \mathrm{~nm}$, i.e., as close as possible to the excitation wavelength. The spectral width of the pump pulse is $\Delta_{\mathrm{Pu}} \approx 6 \mathrm{meV} ; \Delta_{\mathrm{Pu}} \gg \delta_{1}$ ensures that a coherence is actually generated with equivalent initial population of $|X\rangle$ and $|Y\rangle$ sublevels. Beats are observed with a magnitude which represents tens of percents of the global $\Delta T / T$ signal.

Following eq 3 , the optimal contrast in the beating signal is obtained for $\theta=\theta_{0}= \pm \pi / 4$. The beating is visible if $\theta \approx \theta_{0}$ is achieved inside the probed NPLs ensemble, with the further condition that $\theta$ is roughly the same for all the excited NPLs. The disorder in the NPLs orientation, through a distribution of $\theta$, is detrimental to the observation of contrasted oscillations and to the extraction of the key parameters $(\theta>0$ and $\theta<0$ lead to antiphase contributions in the second term of eq 3 ). It is hence unsurprising that the detection of the beating in the differential transmission signal was not systematic but observed only at given positions, in highly diluted samples, where it is very likely that very few NPLs contribute to the signal and where a sufficient degree of local order exists. As a result, we do a

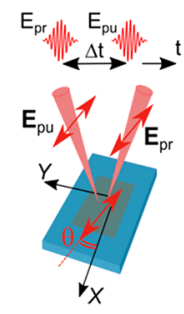

b

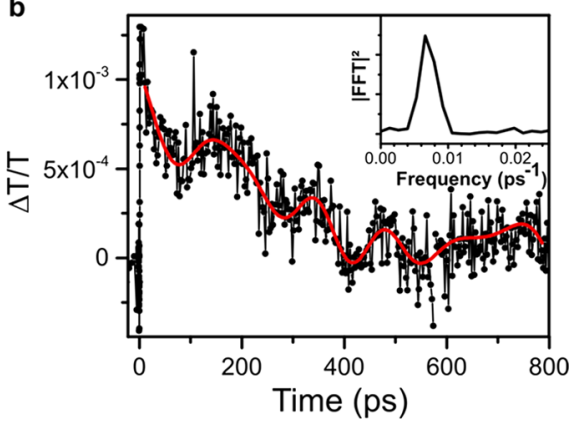

Figure 5. Differential transmittance of highly diluted films in quasiresonant pump-probe configuration. (a) Experimental configuration: the pump $\left(E_{\mathrm{pu}}\right)$ and probe $\left(E_{\mathrm{pr}}\right)$ fields have linear polarizations. The pump is tuned to $\lambda_{\mathrm{pu}} \approx 618 \mathrm{~nm}$, whereas the probe is a broadband continuum $\left(520 \mathrm{~nm}<\lambda_{\mathrm{pr}}<900 \mathrm{~nm}\right.$, delay $\Delta t)$ allowing quasi-degenerate spectral-temporal analysis. The time resolution is $\sim 0.2$ ps. Details of the pump and probe pulse preparation can be found in the Supporting Information. We note $\theta$ as the angle between the incident polarization and the NPL $X$ axis. An analyzer set parallel to the incident polarization is placed on the optical path of the transmitted probe. (b) Differential transmittance signal (solid dots); the solid red line is a guide to the eye. The multistep fitting of the signal using Equation 3 provides the parameters describing the state dynamics $\tau_{\mathrm{R}}, T_{2}$ as well as the EFS parameter, $\delta_{1}$ (see main text and SI). Inset: Fourier transform of the oscillating contribution; the beat period is found to be around $\sim 140 \mathrm{ps}$, leading to a splitting, $\delta_{1} \approx 30 \mu \mathrm{eV}$.

not average eq 3 over $\theta$ and quantify any impact of disorder (both in plane and out of plane). Instead, we regard the experimental decay constant more representative of an inhomogeneous dephasing time, $T_{2}$ *, with the damping of beats very likely strengthened by the remaining inhomogeneity in the EFS that can be described by a distribution of the splitting energies. In light of these assumptions, we shall only insist on the order of magnitude associated with the extracted parameters.

The trace in the inset of Figure 5 represents the Fourier transform of the experimental $\Delta T / T$ signal. The central frequency corresponds to a period $2 \pi / \omega_{1} \approx 140$ ps leading to $\delta_{1} \approx 30 \mu \mathrm{eV}$, in good agreement with the value deduced from the magneto-optical spectroscopy in spite of the simplified basis used for the analysis. We assume here that the states are excited as a coherent superposition and the excitons probed belong to the same inhomogeneous band as investigated by magneto-PL. We thus place the splitting energy value of the studied NPLs in the tens of $\mu \mathrm{eV}$ range. The remaining adjustment provides $T_{2}{ }^{*} \approx 300$ ps as well as $\tau_{\mathrm{R}} \approx 290$ ps which is close to the fastest constant appearing in the emission decay. The values reported for $T_{2}{ }^{*}$ in an ensemble of epitaxial dots shows a rather large variation from $\sim 20$ ps to a few hundreds of ps. ${ }^{46,70,76}$ The fact that the value found in this work is situated in the upper part of the range is particularly important regarding application in quantum devices that are based on coherent manipulations. ${ }^{21}$ We finally note that eq 3 is derived under the assumption that the NPL plane is orthogonal to the polarization plane of the incident light. Here it is also difficult to evaluate the role of NPLs that do not arrange flatwise. NPLs lying on their edge will contribute via stimulated emission from the $|X\rangle($ or $|Y\rangle)$ state, at the rate $\tau_{\mathrm{R}}$ in the differential transmittance signal and will add to the background. We hence conjecture that the orders of magnitude of the parameters 
would not change in the frame of more refined descriptions. In summary, the zero field spectroscopy results outlined also support the assignment of linear polarization within the EFS of indirect excitons.

For type-I dots, either epitaxial or colloidal, a lifting of the $\pm 1\rangle$ state's degeneracy is always accompanied by linear optically active dipoles. ${ }^{38,43}$ The effect is accounted for by the introduction of anisotropy in the electron-hole exchange interaction, itself related to a lowering of the dot symmetry. The latter loss of symmetry can have a more "local" character. For example, symmetry lowering of the crystalline phase in perovskite NCs results in the linearization of states. ${ }^{77,78}$ Nevertheless, the exchange interaction, through its long-range contribution, also probes the shape of the electronic confinement potential and determines states mixing in the EFS. This is accepted as the dominant mechanism for the generation of linearly polarized states in III-V or II-VI semiconductorbased systems with zinc blende structure. ${ }^{30,43}$ In core-crown NPLs, because of the rectangular shape $\left(D_{2}\right.$ symmetry point group), the symmetry axis along the confinement direction is lost which implies states hybridization. ${ }^{38}$ Moreover, the dielectric confinement leads to enhanced Coulombic effects that, in addition to strengthening the binding (IX PL is observed at room temperature), ${ }^{36}$ also contribute to the large exchange interaction responsible for effective $| \pm 1\rangle$ state mixing and large exciton sublevels splittings. Finally, at the present it is hard to estimate the impact of localization at the core-crown interface. In type-I epitaxial QWs although $| \pm 1\rangle$ states are predicted, an important degree of linear polarization is also usually observed. It is related to the contribution of excitons localized in rectangular islands at the QW interface. The state splitting is then due to the in-plane interfacial anisotropy of the localizing potential, a situation that directly compares to the one found in type-I dots. ${ }^{43,79}$ The idea was recently applied to core-shell, type-I, NPLs in order to model the influence of the roughness of the core-shell interface. ${ }^{80}$ In this picture excitonic splitting values resulting from $\approx 1$ monolayer thickness fluctuations seem to be placed in the one $\mathrm{meV}$ to tens of meV range. It could be interesting to adapt this model to the type-II core-crown geometry studied here, taking into account dielectric confinement. We remark that in type-II epitaxial QWs localization models have been abandoned because the exchange interactions have been deemed too small to produce splittings in agreement with experimental observations. Instead models based on hh-lh mixing resulting from symmetry lowering of the interface are preferred. ${ }^{81}$ Nonetheless, the effect of imperfect interfaces and local symmetry effects, despite being challenging to study, warrant further theoretical and numerical investigation to obtain an even clearer view of EFS in core-crown NPLs.

Exciton Spin Relaxation. The time-resolved decay of the DCP is shown in the left panel of Figure 6 for selected magnetic field strengths. Contrary to what was observed for the PL decay, there is no evidence for multiexponential dynamics, further indicating our measurements probe a single relaxation process. We emphasize that competing mechanisms (simultaneous versus sequential carriers spin flip) with distinct dynamics would lead to biexponential decays. ${ }^{82}$ In the following, we identify the mechanism to be the exciton spin flip and extract the exciton spin lifetime, $\tau_{S}$, defined as $\tau_{S}=2 /(\gamma+\bar{\gamma})$ (see model in SI, S9). The plot $\tau_{S}(B)$ is given in the right panel of Figure 6 for the two temperatures, $T=1.6$ and $25 \mathrm{~K}$. At the lowest temperature, $\tau_{\mathrm{S}}(B)$ is perfectly fitted
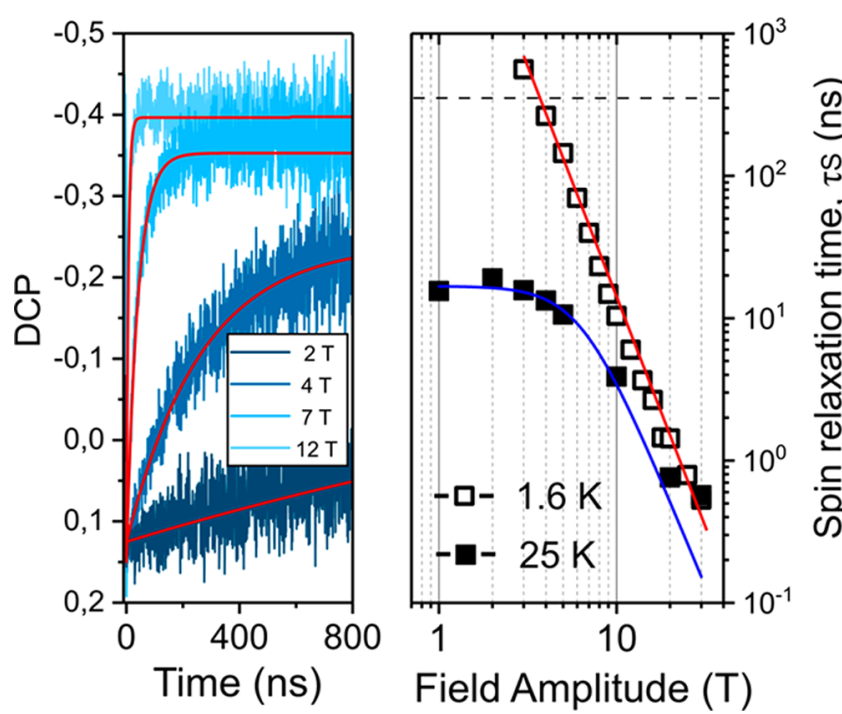

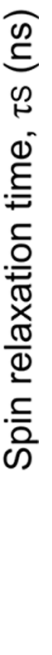

Figure 6. Time-resolved DCP measurements and longitudinal spin relaxation time as a function of the field strength. Left panel: DCP decay kinetics at various magnetic field strengths. DCP curves are extracted from the time-resolved PL (see main text) excited at resonance $\left(\sigma^{+}\right.$excitation, $\left.\lambda_{\text {ex }}=640 \mathrm{~nm}, T=1.6 \mathrm{~K}\right)$. At all magnetic field amplitudes, the curves follow a monoexponential decay. Right panel: longitudinal spin relaxation time (spin lifetime, $\tau_{\mathrm{s}}$ ) as a function of $B$; the values are obtained from the fits of $\operatorname{DCP}(t)$ curves at $T=1.6 \mathrm{~K}$ (open squares) and $25 \mathrm{~K}$ (solid squares). At 1.6 $\mathrm{K}$ reliable lifetimes could not be obtained below $2 \mathrm{~T}$ due to the slow relaxations and noise in data. At high fields $(B>25 \mathrm{~T})$, the spin relaxation time is $\sim 0.5 \mathrm{~ns}$ (setup instrument response $\sim 120$ ps). $\tau_{\mathrm{S}}(B)$ fits to a power law and a modified power law are also plotted as a red solid line and a blue solid line, respectively. The horizontal dashed line marks the value of the exciton lifetime (recombination time) at $1.6 \mathrm{~K}$ (long-lived component of the PL decay).

using a power law: $\tau_{\mathrm{S}}(B) \propto B^{-3.2}$, whereas the curve at $25 \mathrm{~K}$ exhibits a plateau at low field, indicating an interplay of different mechanisms. If we write the relaxation rate, $\Gamma_{\mathrm{S}}=1 /$ $\tau_{\mathrm{S}}$, where $\Gamma_{\mathrm{S}}=\Gamma_{\mathrm{S}, 0}+a B^{-\alpha}$, one consistently finds $\alpha \approx-3.0$ and $\tau_{0}=1 / \Gamma_{\mathrm{S}, 0} \approx 17 \mathrm{~ns}$. On moderate increase from $T \approx 1.6 \mathrm{~K}$, the spin lifetime $\tau_{S}$ quickly decreases by 2 orders of magnitude and crosses the value of $\tau_{\mathrm{R}}$ at $B \approx 3 \mathrm{~T}$ (considering the component $\tau_{i} \geq 350$ ns that contributes the greatest number of emitted photons). We note this is in line with predictions of the model outlined above to explain the inversion of the DCP under $\sigma^{+}$excitation, which roughly occurs around the same field strength.

To the best of our knowledge, investigations of the mechanism of exciton spin-flip have not been made hitherto in type-II nanocrystalline systems. However, some tentative indications can be found in the work of Tsitsishvili et al., ${ }^{47}$ who examined an intrinsic mechanism for spin-flip transitions in asymmetrical dots with classical type-I band structure. Their calculations indeed provide a value for $\alpha=3$. In their scheme the electron and the hole spin flip simultaneously by emitting or absorbing an acoustic phonon. However, the coupling between the degrees of spin and deformations is not contained in the bare electron/hole-phonon Hamiltonian. It is made possible through valence band mixing (between hh and $\mathrm{lh}$ states) due to the interplay of the short-range interaction and the lattice deformation. Hence spin relaxation between $|X\rangle$ and $|Y\rangle$ (and more generally $|E L+\rangle$ and $|E L-\rangle$ states) can be 
envisaged as a result of $\mathrm{lh}-\mathrm{h} h$ exciton mixing that operates as a second order perturbation. However, the model in this work only considers interaction with bulk phonons via a plane-wave phonon field and cannot satisfactorily be applied to freestanding NPLs known to support acoustical modes close to Lamb modes with additional quantization of the phonon wavevector in the confinement direction. Hence, further theoretical studies are required, especially taking into account type-II excitons and the composite structure of the NPL. Let us finally note that the relaxation process should be independent of how the exciton sublevels are initially populated. As expected, a cubic power law in field is indeed observed for the spin relaxation time, at $1.6 \mathrm{~K}$, under nonresonant excitation (see Figure S10).

The $T=25 \mathrm{~K}$ curve joins the $B^{-3}$ asymptote at higher field. At this temperature $k_{\mathrm{B}} T \approx 2.2 \mathrm{meV}$ and corresponds to $B_{0} \approx$ $40 \mathrm{~T}$ in $\delta$. Below $B_{0}$ the separation in energy between the $|E L+\rangle$ and $|E L-\rangle$ levels is lower than the characteristic thermal energy. In this regime $\left(\delta \leq k_{\mathrm{B}} T\right)$ other mechanisms for relaxation should thus be considered. In a similar manner we find $B_{0} \approx 2.3 \mathrm{~T}$ at $T=1.6 \mathrm{~K}$ which roughly sets the limit for the transition at low temperature. Since below $B_{0}$ the DCP becomes quasi-constant across the scanned temporal window, a decay could not be extracted; consequently the low-field region of the curve $(B<2 \mathrm{~T})$ could not be explored within the present work. The weak dependence of $\tau_{\mathrm{S}}$ on the magnetic field below $B_{0}$ does not have a trivial explanation. This could be the signature of multiphonon processes. Contrary to onephonon processes, phonon energies do not need to match the Zeeman energy separation and the remaining field dependence becomes intrinsic to the effective spin-phonon coupling mechanism itself. Theoretical investigations for heavy-hole spin relaxation in quantum dots indeed predict behaviors similar to the one observed in the present work, namely, a saturation of $\tau_{\mathrm{S}}$ at low magnetic fields accompanied by a variation of $\sim 2$ orders of magnitude between $\sim 1$ and $10 \mathrm{~K}^{83}$ However, the theory does not consider the exciton spin-flip mechanism and therefore cannot be directly transposed.

To summarize, $\tau_{S}$ at $2 \mathrm{~T}$ already provides a lower limit of the IX spin lifetime in NPLs ( $\sim 600 \mathrm{~ns})$. As the curve inclination is still not visible, significantly higher values of $\tau_{\mathrm{S}}$ are expected at zero field. The present work allows us to place it in the $\mu \mathrm{s}$ range (at low temperature), much longer than the previously reported value in type-I CdSe/CdS NPLs where $\tau_{\mathrm{S}}$ is found in the ns range, ${ }^{7}$ and comparable to the relaxation time measured in QWs combining indirect band gap and type-II band alignment. $^{84}$

\section{CONCLUSIONS}

In this work, the EFS of indirect (or charge transfer) excitons in type-II core-crown CdSe/CdTe NPLs has been studied using low-temperature magneto-optical techniques under high fields and nonlinear optical spectroscopy at zero field. Quasiresonant excitation of the IX transition, using $\sigma^{+}$and $\sigma^{-}$ polarized light, allows the selective addressing of the fine structure sublevels. DCP and DLP measurements allow us to circumvent the issue of strong inhomogeneous broadening of the IX transition and to evidence the two optically active, linearly polarized states that compose the EFS at zero field. The model developed to account for the DCP and DLP measurements leads to the values of the IX Landé factor $\left(g_{z} \approx\right.$ 0.7 ) and to the zero-field energy splitting, $\delta_{1}$, between the evidenced bright levels (found in the tens of $\mu \mathrm{eV}$ range). This first determination of $\delta_{1}$ is consistent with the results from the quantum-beat experiments performed in the pump-probe configuration where we monitor the time evolution of a pumpinduced coherent superposition of the EFS bright states.

The fact that $\delta_{1}$ compares with the splitting usually observed in type-I epitaxial dots or type-I NCs, presenting a similar degree of confinement, is a consequence of the strong dielectric contrast that IXs experience in NPLs and explains the intriguingly strong IX binding. On this basis, we conjecture that despite the type-II band alignment, the mixing of the states of momentum $M_{s}= \pm 1$ leading to linear dipolar activity, is due to the anisotropy of the electron-hole exchange interaction arising from the breaking of NPLs in-plane rotational symmetry. We also show that relaxation between fine structure sublevels is most likely driven by the direct spinflip process between the bright sublevels. Additionally, we provide an estimate for a lower bound of the exciton spin lifetime at low temperature, $\tau_{S} \approx 1 \mu \mathrm{s}$. An evaluation of the spin coherence properties is also obtained with the estimation of the inhomogeneous dephasing time from the beating experiment $\left(T_{2}{ }^{*} \approx 300 \mathrm{ps}\right)$. These results all indicate the strong potential of such NPLs to retain spin information and be of promise for quantum devices. Several questions are raised at the same time by this work such as the origin of the $B^{-3}$ dependence of the exciton "direct" spin-flip rate (evidenced at low temperature), the nature of the spin relaxation mechanisms that take over at higher temperatures and the apparent absence of effects that would be the signature of coupling between bright and dark excitons. In particular, the precise positioning of the IX dark state and the magnitude of the bright-dark state coupling, remain important questions. Further experimental and theoretical investigations are required to examine the origin of these effects.

\section{EXPERIMENTAL METHODS}

Sample preparation. Four monolayer thick core-crown CdSe/ $\mathrm{CdTe}$ nanoplatelets were prepared as detailed by Pedetti et al. ${ }^{15}$ (see SI, S1, for full details). NPL films were prepared on precleaned (acetone/2-propanol/ $\mathrm{O}_{2}$ plasma) $4 \mathrm{~mm} \times 4 \mathrm{~mm}$ quartz substrates. NPLs in hexane $(25 \mathrm{mg} / \mathrm{mL})$ were mixed in a $1: 2$ ratio with polystyrene $\left(250,000 M_{w}\right.$; Sigma) in toluene. The solution was then dropcasted or doctor bladed (at room temperature) onto the glass substrates to produce homogeneous films. A second set of samples where no polystyrene was added were also prepared. The magnetic field response of such platelets was found to be identical. Samples preparation was performed in an inert Ar atmosphere to prevent oxidative damage; samples were vacuum sealed for transport. All measurements were performed within 2 weeks of nanoplatelet synthesis with a minimum exposure of platelets to the environment.

Temperature-Dependent Absorption. An Agilent Cary 6000i UV-vis-NIR spectrophotometer with blank substrate correction was used. Spin-coated samples on fused silica substrates were placed in a continuous-flow cryostat (Oxford Instruments Optistat CF-V) under a helium atmosphere. We allowed the sample temperature to equilibrate for $30 \mathrm{~min}$ before taking data.

Magneto-Circular Dichroism Spectroscopy. Magneto-circular dichroism measurements were performed on a $\mathrm{CD}$ spectrometer (J$1500 \mathrm{CD}$, JASCO corporation) using 0.4 and $1.6 \mathrm{~T}$ removable permanent magnets (JASCO PM-409 and PM-491, respectively). Samples were measured as dilute dispersions of hexane solution with an approximate sample OD of 0.6 at $550 \mathrm{~nm}(1 \mathrm{~mm}$ path length cuvettes (Helma)). The scan rate, resolution and data interval time were set to $50 \mathrm{~nm} / \mathrm{min}, 1 \mathrm{~nm}$ and $1 \mathrm{~s}$, respectively. To obtain accurate MCD devoid of any artifacts from the selective reflection and scattering, spectra were recorded at two magnet orientations, $\mathrm{N} \rightarrow \mathrm{S}$ (north to south) and $\mathrm{S} \rightarrow \mathrm{N}$ (south to north), in the direction of 
propagation of incident light. The MCD signal was then taken as the difference between the two spectra divided by two: $\mathrm{MCD}=\frac{\mathrm{CD}(\mathrm{N} \rightarrow S)-\mathrm{CD}(\mathrm{S} \rightarrow \mathrm{N})}{2}$.

Degree of Linear Polarization Measurements at Zero Magnetic Field. The DLP measurements at zero magnetic field were performed on NPLs mixed with polystyrene and dropcasted onto $4 \mathrm{~mm} \times 4 \mathrm{~mm}$ glass slides. The samples were mounted in a cryostat (Oxford Instruments) and cooled to $5 \mathrm{~K}$. The excitation, delivered by an optical parametric oscillator $(82 \mathrm{MHz}$ repetition rate), was tuned at $640 \mathrm{~nm}$; the spot size of the beam at the focus was $\sim 5$ $\mu \mathrm{m}$. The polarization of the excitation was controlled by a thin film polarizer followed by a half-wave plate along the optical path. The PL of the NPLs was collected with a $0.25 \mathrm{NA}$ objective and the polarization of the PL was directly analyzed after the objective with a motorized half-wave plate (Thorlabs) and an analyzer (the direction of which is parallel to the grating grooves of the spectrometer). A Semrock filter was used to get rid of the scattered light from the excitation and to select the wavelengths of interest in the spectra.

Steady-State and Time-Resolved Photoluminescence Spectroscopy with High Magnetic Fields. Measurements were performed at the High Field Magnet Laboratory, Nijmegen, Netherlands. Samples were mounted in a titanium holder and mounted onto an $x y z$ piezo stage (AttoCube). The stage was placed in an optical probe and mounted inside a liquid helium bath cryostat $(4.2 \mathrm{~K})$ and inserted in a $50 \mathrm{~mm}$ bore Florida-Bitter electromagnet with a maximum DC magnetic field strength of $31 \mathrm{~T}$. Measurements performed at $1.6 \mathrm{~K}$ were carried out under pumped Helium. Experiments were performed in Faraday geometry with the excitation and detection light parallel to the magnetic field direction. Picosecond laser diodes (PicoQuant) operating in either c.w. or pulsed mode $(250 \mathrm{kHz})$ and centered either at 485 and $640 \mathrm{~nm}$ were used to excite the sample. Spatially filtered laser light was focused onto the sample using a 1.1 NA objective with PL collected through the objective in a backscattering geometry. The excitation light was filtered using appropriate long-pass filters (Semrock). After passing through a calibrated waveplate (Melles Griot) and polarizer (Thorlabs) combination, the PL was focused with a $150 \mathrm{~mm}$ lens onto either the slit of a Princeton Instruments Si CCD spectrometer (1200 lines per mm grating) or Si avalanche photodiode (MPD) connected to a single-photon counter. The time resolution of the time-correlated single-photon counting setup was approximately $\sim 120 \mathrm{ps}$. The sample temperature was left to equilibrate for at least $30 \mathrm{~min}$ prior to measurement. Further details of the measurement are provided in the Supporting Information (S1). Subnanosecond time-resolved PL experiments (overall time resolution $\sim 10 \mathrm{ps)}$ ) were performed using a streak camera (C5680 model from Hamamatsu) coupled to a Acton SP2760i imaging spectrometer (focal length $f=750 \mathrm{~mm}, 300$ lines/ $\mathrm{mm}$ grating, Princeton Instrument).

Picosecond Spin Beating Experiments. The ps-spin beating TA experiments were performed using an $\mathrm{Yb}$-based amplified system (PHAROS, Light Conversion) providing $14.5 \mathrm{~W}$ at $1030 \mathrm{~nm}$ and 38 $\mathrm{kHz}$ repetition rate. The probe beam is generated by focusing a portion of the fundamental into a $4 \mathrm{~mm}$ YAG substrate and spans from 520 to $900 \mathrm{~nm}$. The pump beam is generated by seeding a portion of the fundamental to a narrow band optical parametric oscillator (ORPHEUS-LYRA, Light Conversion). The pump pulse was set to $618 \mathrm{~nm}$ and narrowed to an fwhm of $\sim 2 \mathrm{~nm}(6 \mathrm{meV})$ by means of short-pass-band-pass filter combination (Semrock). Thin films of dilute NPLs were prepared by mixing a $0.01 \mathrm{mg} / \mathrm{mL}$ solution of CdSe/CdTe NPLs with $4 \mathrm{~mL}$ of toluene/polystyrene $\left(200,000 M_{\mathrm{w}}\right.$; Sigma). The resulting solution was blade coated onto quartz slides (Spectrosil) to produce homogeneous thin films. Samples were placed in a cryostat (Janis Instruments) and cooled down to $6 \mathrm{~K}$. The sample temperature was left to equilibrate for $90 \mathrm{~min}$ prior to measuring. The pump and probe beams were overlapped and focused onto the sample; there are approximately 50-100 NPLs estimated to be within the excitation spot. The NPLs are assumed to be approximately randomly orientated within the film. The pump and probe pulse polarizations were set to the same linear polarization by means of a half-waveplate (Thorlabs), i.e., parallel-parallel pump probe configuration. The pump fluence was typically $45 \mu \mathrm{J} / \mathrm{cm}^{2}$. The white light probe was delayed using a computer-controlled piezoelectric translation stage (Newport), and a sequence of probe pulses with and without pump was generated using a chopper wheel (Thorlabs) on the pump beam. The linear polarization of the probe pulse transmitted through the sample was detected by a silicon photodiode array camera (Stressing Entwicklunsbüro).

Data Analysis. Data analysis was carried out with custom codes written using MATLAB and Origin software. The details of specific algorithms used in the analysis are discussed in the Supporting Information.

\section{ASSOCIATED CONTENT}

\section{Supporting Information}

The Supporting Information is available free of charge on the ACS Publications website at DOI: 10.1021/acsnano.9b03252.

Sample preparation, experimental setups for high field and ps-spin beating experiments, ultramicrotomy TEM images, MCD measurements and calculations, $g$-factor sign calibration, temperature dependence of DCP, spin relaxation under nonresonant $(480 \mathrm{~nm})$ excitation, theoretical modeling of orientation effects on DCP and spin relaxation, DLP measurements and model, and calculations on the role of dielectric confinement (PDF)

\section{AUTHOR INFORMATION}

\section{Corresponding Author}

*E-mail: barisien@insp.jussieu.fr.

ORCID $\odot$

Raj Pandya: 0000-0003-1108-9322

Yuttapoom Puttisong: 0000-0002-9690-6231

Marion Dufour: 0000-0001-9588-9524

Thierry Barisien: 0000-0003-1814-5948

Girish Lakhwani: 0000-0003-1070-5859

Sandrine Ithurria: 0000-0002-4733-9883

Akshay Rao: 0000-0003-4261-0766

\section{Notes}

The authors declare no competing financial interest.

The raw data associated with the manuscript is available at https://doi.org/10.17863/CAM.43445.

\section{ACKNOWLEDGMENTS}

R.P. and A.R. acknowledge the EPSRC (UK) and the Winton Program for the Physics of Sustainability for financial support. R.P. thanks Emrys Evans, Leah Weiss, Hannah Stern, Murad Tayebjee, Alexander Cheminal, Simon Dowland, Tudor H. Thomas, and Neil C. Greenham (Cambridge) for useful advice, discussions, and assistance with preliminary experiments as well as Giorgio Divitini for ultramicrotomy TEM images. Y.P. and W.M.C. acknowledge support from the Swedish Research Council (Grant Nos. 2017-05285 and 201605091). V.S., T.B., L.L., M.C., C.T., and F.B. warmly thank Mathieu Bernard and Maxime Vabre for their technical assistance at INSP in cryogenics and Florent Margaillan for optics. A.S. and G.L. thank the Australian Research Council for support under Grant No. CE170100026. This work was supported by HFML-RU/NOW-I, member of the European Magnetic Field Laboratory (EMFL) and by EPSRC (UK) via its membership to the EMFL (Grant No. EP/N01085X/1). We thank Juan Climente (Universitat Jaume I, Spain) for 
invaluable discussions during the preparation of this manuscript.

\section{REFERENCES}

(1) Sweeney, S. J.; Mukherjee, J. Optoelectronic Devices and Materials. In Springer Handbook of Electronic and Photonic Materials; Kasap, S., Capper, P., Eds; Springer: Cham, 2017; pp 897-932.

(2) Brennan, K. F.; Brown, A. S. Semiconductor Heterostructures. In Theory of Modern Electronic Semiconductor Devices; John Wiley and Sons, Inc.: New York, 2002; pp 14-83.

(3) Ithurria, S.; Tessier, M. D.; Mahler, B.; Lobo, R. P. S. M.; Dubertret, B.; Efros, A. L. Colloidal Nanoplatelets with TwoDimensional Electronic Structure. Nat. Mater. 2011, 10, 936-941.

(4) Kelestemur, Y.; Guzelturk, B.; Erdem, O.; Olutas, M.; Erdem, T.; Usanmaz, C. F.; Gungor, K.; Demir, H. V. CdSe/CdSe ${ }_{1-x} \mathrm{Te}_{x}$ Core/ Crown Heteronanoplatelets: Tuning the Excitonic Properties without Changing the Thickness. J. Phys. Chem. C 2017, 121, 4650-4658.

(5) Rajadell, F.; Climente, J. I.; Planelles, J. Excitons in Core-Only, Core-Shell and Core-Crown CdSe Nanoplatelets: Interplay Between In-Plane Electron-Hole Correlation, Spatial Confinement, and Dielectric Confinement. Phys. Rev. B: Condens. Matter Mater. Phys. 2017, 96, 035307.

(6) Shornikova, E. V.; Biadala, L.; Yakovlev, D. R.; Sapega, V.; Kusrayev, Y. G.; Mitioglu, A. A.; Ballottin, M. V.; Christianen, P. C. M.; Belykh, V. V.; Kochiev, M. V.; Sibeldin, N. N.; Golovatenko, A. A.; Rodina, A. V.; Gippius, N. A.; Kuntzmann, A.; Jiang, Y.; Nasilowski, M.; Dubertret, B.; Bayer, M. Addressing the Exciton Fine Structure in Colloidal Nanocrystals: the Case of CdSe Nanoplatelets. Nanoscale 2018, 10, 646-656.

(7) Shornikova, E. V.; Biadala, L.; Yakovlev, D. R.; Feng, D.; Sapega, V. F.; Flipo, N.; Golovatenko, A. A.; Semina, M. A.; Rodina, A. V.; Mitioglu, A. A.; Ballottin, M. V.; Christianen, P. C. M.; Kusrayev, Y. G.; Nasilowski, M.; Dubertret, B.; Bayer, M. Electron and Hole gFactors and Spin Dynamics of Negatively Charged Excitons in CdSe/ CdS Colloidal Nanoplatelets with Thick Shells. Nano Lett. 2018, 18, 373-380.

(8) Scott, R.; Kickhöfel, S.; Schoeps, O.; Antanovich, A.; Prudnikau, A.; Chuvilin, A.; Woggon, U.; Artemyev, M.; Achtstein, A. W. Temperature Dependent Radiative and Non-Radiative Recombination Dynamics in $\mathrm{CdSe}-\mathrm{CdTe}$ and $\mathrm{CdTe}-\mathrm{CdSe}$ Type II Hetero Nanoplatelets. Phys. Chem. Chem. Phys. 2016, 18, 3197-3203.

(9) Cassette, E.; Pedetti, S.; Mahler, B.; Ithurria, S.; Dubertret, B.; Scholes, G. D. Ultrafast Exciton Dynamics in 2D In-Plane HeteroNanostructures: Delocalization and Charge Transfer. Phys. Chem. Chem. Phys. 2017, 19, 8373-8379.

(10) Dede, D.; Taghipour, N.; Quliyeva, U.; Sak, M.; Kelestemur, Y.; Gungor, K.; Demir, H. V. Highly Stable Multicrown Heterostructures of Type II Nanoplatelets for Ultralow Threshold Optical Gain. Chem. Mater. 2019, 31, 1818-1826.

(11) Wu, K.; Li, Q.; Jia, Y.; McBride, J. R.; Xie, Z. X.; Lian, T. Efficient and Ultrafast Formation of Long-Lived Charge-Transfer Exciton State in Atomically Thin Cadmium Selenide/Cadmium Telluride Type-II Heteronanosheets. ACS Nano 2015, 9, 961-968.

(12) Li, Q.; Xu, Z.; McBride, J. R.; Lian, T. Low Threshold Multiexciton Optical Gain in Colloidal CdSe/CdTe Core/Crown Type-II Nanoplatelet Heterostructures. ACS Nano 2017, 11, 25452553.

(13) Olutas, M.; Guzelturk, B.; Kelestemur, Y.; Yeltik, A.; Delikanli, S.; Demir, H. V. Lateral Size-Dependent Spontaneous and Stimulated Emission Properties in Colloidal CdSe Nanoplatelets. ACS Nano 2015, 9, 5041-5050.

(14) Kelestemur, Y.; Olutas, M.; Delikanli, S.; Guzelturk, B.; Akgul, M. Z.; Demir, H. V. Type-II Colloidal Quantum Wells: CdSe/CdTe Core/Crown Heteronanoplatelets. J. Phys. Chem. C 2015, 119, 21772185.

(15) Pedetti, S.; Ithurria, S.; Heuclin, H.; Patriarche, G.; Dubertret, B. Type-II CdSe/CdTe Core/Crown Semiconductor Nanoplatelets. J. Am. Chem. Soc. 2014, 136, 16430-16438.
(16) Novoselov, K. S.; Mishchenko, A.; Carvalho, A.; Castro Neto, A. H. 2D Materials and van der Waals Heterostructures. Science 2016, $353,9439$.

(17) Jariwala, D.; Marks, T. J.; Hersam, M. C. Mixed-Dimensional van der Waals Heterostructures. Nat. Mater. 2017, 16, 170-181.

(18) Meinardi, F.; Colombo, A.; Velizhanin, K. A.; Simonutti, R.; Lorenzon, M.; Beverina, L.; Viswanatha, R.; Klimov, V. I.; Brovelli, S. Large-Area Luminescent Solar Concentrator Based on 'Stokes-ShiftEngineered' Nanocrystals in a Mass-Polymerized PMMA Matrix. Nat. Nat. Photonics 2014, 8, 392-399.

(19) Sigle, D. O.; Zhang, L.; Ithurria, S.; Dubertret, B.; Baumberg, J. J. Ultrathin CdSe in Plasmonic Nanogaps for Enhanced Photocatalytic Water Splitting. J. Phys. Chem. Lett. 2015, 6, 1099-1103.

(20) Taherkhani, M.; Willatzen, M.; Mørk, J.; Gregersen, N.; McCutcheon, D. P. S. Type-II Quantum-Dot-in-Nanowire Structures with Large Oscillator Strength for Optical Quantum Gate Applications. Phys. Rev. B: Condens. Matter Mater. Phys. 2017, 96, 125408 .

(21) Ortner, G.; Bayer, B.; Lyanda-Geller, Y.; Reinecke, T. L.; Kress, A.; Reithmaier, J. P.; Forchel, A. Control of Vertically Coupled InGaAs/GaAs Quantum Dots with Electric Fields. Phys. Rev. Lett. 2005, 94, 157401.

(22) Klenovsky, P.; Steindl, P.; Geffroy, D. Excitonic Structure and Pumping Power Dependent Emission Blue-Shift of Type-II Quantum Dots. Sci. Rep. 2017, 7, 45568.

(23) Benson, O.; Santori, C.; Pelton, M.; Yamamoto, Y. Regulated and Entangled Photons from a Single Quantum Dot. Phys. Rev. Lett. 2000, 84, 2513-2516.

(24) Stevenson, R. M.; Hudson, A. J.; Bennett, A. J.; Young, R. J.; Nicoll, C. A.; Ritchie, D. A.; Shields, A. J. Evolution of Entanglement Between Distinguishable Light States. Phys. Rev. Lett. 2008, 101, 170501 .

(25) Winik, R.; Cogan, D.; Don, Y.; Schwartz, I.; Gantz, L.; Schmidgall, E. R.; Livneh, N.; Rapaport, R.; Buks, E.; Gershoni, D. On-Demand Source of Maximally Entangled Photon Pairs Using the Biexciton-Exciton Radiative Cascade. Phys. Rev. B: Condens. Matter Mater. Phys. 2017, 95, 235435.

(26) Ma, X.; Diroll, B. T.; Cho, W.; Fedin, I.; Schaller, R. D.; Talapin, D. V.; Gray, S. K.; Wiederrecht, G. P.; Gosztola, D. J. SizeDependent Biexciton Quantum Yields and Carrier Dynamics of Quasi-Two-Dimensional Core/Shell Nanoplatelets. ACS Nano 2017, 11, 9119-9127.

(27) Wegner, K. D.; Hildebrandt, N. Quantum Dots: Bright and Versatile In Vitro and In Vivo Fluorescence Imaging Biosensors. Chem. Soc. Rev. 2015, 44, 4792-4834.

(28) Ko, Y. H.; Jalalah, M.; Lee, S. J.; Park, J. G. Super Ultra-High Resolution Liquid-Crystal-Display Using Perovskite Quantum Dot Functional Color-Filters. Sci. Rep. 2018, 8, 12881.

(29) Baskoutas, S.; Jia, Y.; Zeng, Z.; Bester, G.; Garoufalis, C. S. Realization of Linearly Polarized Exciton Emission in Wurtzite Zinc Oxide Quantum Dots. Phys. Rev. B: Condens. Matter Mater. Phys. 2018, 98, 235410.

(30) Htoon, H.; Furis, M.; Crooker, S. A.; Jeong, S.; Klimov, V. I. Linearly Polarized 'Fine Structure' of the Bright Exciton State in Individual CdSe Nanocrystal Quantum Dots. Phys. Rev. B: Condens. Matter Mater. Phys. 2008, 77, 035328.

(31) Pandya, R.; Chen, R. Y. S.; Cheminal, A.; Dufour, M.; Richter, J. M.; Thomas, T. H.; Ahmed, S.; Sadhanala, A.; Booker, E. P.; Divitini, G.; Deschler, F.; Greenham, N. C.; Ithurria, S.; Rao, A. Exciton-Phonon Interactions Govern Charge-Transfer-State Dynamics in CdSe/CdTe Two-Dimensional Colloidal Heterostructures. J. Am. Chem. Soc. 2018, 140, 14097-14111.

(32) Kuno, M.; Nirmal, M.; Bawendi, M. G.; Efros, A.; Rosen, M. Magnetic Circular Dichroism Study of CdSe Quantum Dots. J. Chem. Phys. 1998, 108, 4242.

(33) Gromova, Y. A.; Miropoltsev, M. A.; Cherevkov, S. A.; Maslov, V. G.; Baranov, A. V.; Fedorov, A. V. Magnetic Circular Dichroism in 2D Colloidal Semiconductor Nanocrystals. Opt. Spectrosc. 2018, 125, 698-702. 
(34) Beaulac, R.; Ochsenbein, S. T.; Gamelin, D. R. Colloidal Transition-Metal-Doped Quantum Dots. In Nanocrystal Quantum Dots, 2nd ed.; Klimov, V. I., Ed.; CRC Press: Boca Raton, FL, 2010; pp 397-453.

(35) Tessier, M. D.; Javaux, C.; Maksimovic, I.; Loriette, V.; Dubertret, B. Spectroscopy of Single CdSe Nanoplatelets. ACS Nano 2012, 6, 6751-6758.

(36) Dufour, M.; Steinmetz, V.; Izquierdo, E.; Pons, T.; Lequeux, N.; Lhuillier, E.; Legrand, L.; Chamarro, M.; Barisien, T.; Ithurria, S. Engineering Bicolor Emission in 2D Core/Crown CdSe/CdSe ${ }_{1-x} \mathrm{Te}_{x}$ Nanoplatelet Heterostructures Using Band-Offset Tuning. J. Phys. Chem. C 2017, 121, 24816-24823.

(37) Granados Del Águila, A.; Jha, B.; Pietra, F.; Groeneveld, E.; De Mello Donegá, C.; Maan, J. C.; Vanmaekelbergh, D.; Christianen, C. Observation of the Full Exciton and Phonon Fine Structure in CdSe/ CdS Dot-in-Rod Heteronanocrystals. ACS Nano 2014, 8, 5921-5931.

(38) Bayer, M.; Ortner, G.; Stern, O.; Kuther, A.; Gorbunov, A. A.; Forchel, A.; Hawrylak, P.; Fafard, S.; Hinzer, K.; Reinecke, T. L.; Walck, S. N.; Reithmaier, J. P.; Klopf, F.; Schäfer, F. Fine Structure of Neutral and Charged Excitons in Self-Assembled In(Ga)As/(Al)GaAs Quantum Dots. Phys. Rev. B: Condens. Matter Mater. Phys. 2002, 65, 195315.

(39) Marie, X.; Urbaszek, B.; Krebs, O.; Amand, T. In Spin Physics in Semiconductors; Dyakonov, M. I., Ed.; Springer Series in Solid-State Sciences; Springer-Verlag: Berlin, 2008; Vol. 157 pp 105-129.

(40) Kusrayev, Y. G. Optical Orientation of Excitons and Carriers in Quantum Dots. Semicond. Sci. Technol. 2008, 23, 114013.

(41) Yakovlev, D. R.; Platonov, A. V.; Ivchenko, E. L.; Kochereshko, V. P.; Sas, C.; Ossau, W.; Hansen, L.; Waag, A.; Landwehr, G.; Molenkemp, L. W. Hidden In-Plane Anisotropy of Interfaces in $\mathrm{Zn}(\mathrm{Mn}) \mathrm{Se} / \mathrm{BeTe}$ Quantum Wells with a Type-II Band Alignment. Phys. Rev. Lett. 2002, 88, 257401.

(42) Johnston-Halperin, E.; Awschalom, D. D.; Crooker, S. A.; Efros, Al.L.; Rosen, M.; Peng, X.; Alivisatos, A. P. Spin Spectroscopy of Dark Excitons in CdSe Quantum Dots to 60 T. Phys. Rev. B: Condens. Matter Mater. Phys. 2001, 63, 205309.

(43) Ivchenko, E. L. Fine Structure of Excitonic Levels in Semiconductor Nanostructures. Phys. Stat. Sol.(a) 1997, 164, 487492.

(44) Gammon, D.; Snow, E. S.; Shanabrook, B. V.; Katzer, D. S.; Park, D. Fine Structure Splitting in the Optical Spectra of Single GaAs Quantum Dots. Phys. Rev. Lett. 1996, 76, 3005-3008.

(45) Krizhanovskii, D. N.; Ebbens, A.; Tartakovskii, A. I.; Pulizzi, F.; Wright, T.; Skolnick, M. S.; Hopkinson, M. Individual Neutral and Charged InxGa1-xAs-GaAs Quantum Dots with Strong In-Plane Optical Anisotropy. Phys. Rev. B: Condens. Matter Mater. Phys. 2005, 72, No. 161312

(46) Siarry, B.; Eble, B.; Bernardot, F.; Grinberg, P.; Testelin, T.; Chamarro, M.; Lemaitre, A. Magnetic-Field Control of the Exciton Quantum Beats Phase in InGaAs/GaAs Quantum Dots. Phys. Rev. B: Condens. Matter Mater. Phys. 2015, 92, 155315.

(47) Tsitsishvili, E.; Baltz, R. V.; Kalt, H. Exciton Spin Relaxation in Single Semiconductor Quantum Dots. Phys. Rev. B: Condens. Matter Mater. Phys. 2003, 67, 205330.

(48) Khaetskii, A. V.; Nazarov, Y. V. Spin-Flip Transitions Between Zeeman Sublevels in Semiconductor Quantum Dots. Phys. Rev. B: Condens. Matter Mater. Phys. 2001, 64, 125316.

(49) Sénès, M.; Urbaszek, B.; Marie, X.; Amand, T.; Tribollet, J.; Bernardot, F.; Testelin, C.; Chamarro, M.; Gérard, J.-M. Exciton Spin Manipulation in InAs/GaAs Quantum Dots: Exchange Interaction and Magnetic Field Effects. Phys. Rev. B: Condens. Matter Mater. Phys. 2005, 71, 115334.

(50) Léger, Y.; Besombes, L.; Maingault, L.; Mariette, H. ValenceBand Mixing in Neutral, Charged, and Mn-Doped Self-Assembled Quantum Dots. Phys. Rev. B: Condens. Matter Mater. Phys. 2007, 76, 045331 .

(51) Furis, M.; Htoon, H.; Petruska, M. A.; Klimov, V.; Barrick, T.; Crooker, S. A. Bright-Exciton Fine Structure and Anisotropic
Exchange in CdSe Nanocrystal Quantum Dots. Phys. Rev. B: Condens. Matter Mater. Phys. 2006, 73, 241313.

(52) Kumagai, M.; Takagahara, T. Excitonic and Nonlinear-Optical Properties of Dielectric Quantum-Well Structures. Phys. Rev. B: Condens. Matter Mater. Phys. 1989, 40, 12359-12381.

(53) Rodina, A. V.; Efros, Al.L Effect of Dielectric Confinement on Optical Properties of Colloidal Nanostructures. J. Exp. Theor. Phys. 2016, 122, 554-566.

(54) Benchamekh, R.; Gippius, N. A.; Even, J.; Nestoklon, M. O.; Jancu, J.-M.; Ithurria, S.; Dubertret, B.; Efros, Al.L Tight-Binding Calculations of Image-Charge Effects in Colloidal Nanoscale Platelets of CdSe. Phys. Rev. B: Condens. Matter Mater. Phys. 2014, 89, 035307.

(55) Lelong, Ph.; Suzuki, K.; Bastard, G.; Sakaki, H.; Arakawa, Y. Enhancement of the Coulomb Correlations in Type-II Quantum Dots. Phys. E 2000, 7, 393-397.

(56) Matsuda, K.; Nair, S. V.; Ruda, H. E.; Sugimoto, Y.; Saiki, T.; Yamaguchi, K. Two-Exciton State in GaSb/GaAs Type II Quantum Dots Studied Using Near-Field Photoluminescence Spectroscopy. Appl. Phys. Lett. 2007, 90, 013101.

(57) De Godoy, M. P. F.; Gomes, P. F.; Nakaema, M. K. K.; Iikawa, F.; Brasil, M. J. S. P.; Caetano, R. A.; Madureira, J. R.; Bortoleto, J. R. R.; Cotta, M. A.; Ribeiro, E.; Marques, G. E.; Bittencourt, A. C. R. Exciton g Factor of Type-II InP/GaAs Single Quantum Dots. Phys. Rev. B: Condens. Matter Mater. Phys. 2006, 73, 033309.

(58) Piryatinski, A.; Ivanov, S. A.; Tretiak, S.; Klimov, V. I. Effect of Quantum and Dielectric Confinement on the Exciton-Exciton Interaction Energy in Type II Core/Shell Semiconductor Nanocrystals. Nano Lett. 2007, 7, 108-115.

(59) Wang, S.; Wang, L.-W. Exciton Dissociation in CdSe/CdTe Heterostructure Nanorods. J. Phys. Chem. Lett. 2011, 2, 1-6.

(60) Krapek, V.; Klenovsky, P.; Sikola, T. Excitonic Fine Structure Splitting in Type-II Quantum Dots. Phys. Rev. B: Condens. Matter Mater. Phys. 2015, 92, 195430.

(61) Rabouw, F. T.; Van Der Bok, J. C.; Spinicelli, P.; Mahler, B.; Nasilowski, M.; Pedetti, S.; Dubertret, B.; Vanmaekelbergh, D. Temporary Charge Carrier Separation Dominates the Photoluminescence Decay Dynamics of Colloidal CdSe Nanoplatelets. Nano Lett. 2016, 16, 2047-2053.

(62) Granados del Águila, A.; Groeneveld, E.; Maan, J. C.; De Mello Donegá, C.; Christianen, P. C. M. Effect of Electron-Hole Overlap and Exchange Interaction on Exciton Radiative Lifetimes of CdTe/ CdSe Heteronanocrystals. ACS Nano 2016, 10, 4102-4110.

(63) Lee, W.; Park, S.; Murayama, A.; Lee, J.-S.; Kyhm, K. Magnetic Field Insensitive Photoluminescence Decay of $\mathrm{ZnSe} / \mathrm{CdS}$ Core/Shell Type-II Colloidal Quantum Dots. Jpn. J. Appl. Phys. 2018, 57, 06HE06.

(64) Liu, F.; Biadala, L.; Rodina, V.; Yakovlev, D. R.; Dunker, D.; Javaux, C.; Hermier, J.-P.; Efros, A. L.; Dubertret, B.; Bayer, M. Spin Dynamics of Negatively Charged Excitons in CdSe/CdS Colloidal Nanocrystals. Phys. Rev. B: Condens. Matter Mater. Phys. 2013, 88, 035302 .

(65) Labeau, O.; Tamarat, P.; Lounis, B. Temperature Dependence of the Luminescence Lifetime of Single CdSe/ZnS Quantum Dots. Phys. Rev. Lett. 2003, 90, 257404.

(66) Royo, M.; Climente, J. I.; Movilla, J. L.; Planelles, J. Dielectric Confinement of Excitons in Type-I and Type-II Semiconductor Nanorods. J. Phys.: Condens. Matter 2011, 23, 015301.

(67) Beian, M.; Alloing, M.; Cambril, E.; Gomez Carbonell, C.; Osmond, J.; Lemaitre, A.; Dubin, F. Long-Lived Spin Coherence of Indirect Excitons in GaAs Coupled Quantum Wells. EPL 2015, 110, 27001.

(68) Kowalik-Seidl, K.; Vögele, X. P.; Rimpfl, B. N.; Manus, S.; Kotthaus, J. P.; Schuh, D.; Wegscheider, W.; Holleitner, A. W. Long Exciton Spin Relaxation in Coupled Quantum Wells. Appl. Phys. Lett. 2010, 97, 011104.

(69) Fernée, M. J.; Sinito, C.; Louyer, Y.; Potzner, C.; Nguyen, T.-L.; Mulvaney, P.; Tamarat, P.; Lounis, B. Magneto-Optical Properties of Trions in Non-Blinking Charged Nanocrystals Reveal an Acoustic Phonon Bottleneck. Nat. Commun. 2012, 3, 1287. 
(70) Lenihan, A. S.; Gurudev Dutt, M. V.; Steel, D. G.; Ghosh, S.; Bhattacharya, P. K. Raman Coherence Beats from Entangled Polarization Eigenstates in InAs Quantum Dots. Phys. Rev. Lett. 2002, 88, 223601.

(71) Tartakovskii, A. I.; Makhonin, M. N.; Sellers, I. R.; Cahill, J.; Andreev, A. D.; Whittaker, D. M.; Wells, J.-P. R.; Fox, A. M.; Mowbray, D. J.; Skolnick, M. S.; Groom, K. M.; Steer, M. J.; Liu, H. Y.; Hopkinson, M. Effect of Thermal Annealing and Strain Engineering on the Fine Structure of Quantum Dot Excitons. Phys. Rev. B: Condens. Matter Mater. Phys. 2004, 70, 193303.

(72) Bar-Ad, S.; Bar-Joseph, I. Absorption Quantum Beats of Magnetoexcitons in GaAs Heterostructures. Phys. Rev. Lett. 1991, 66, 2491-2494.

(73) Bernardot, F.; Aubry, E.; Tribollet, J.; Testelin, C.; Chamarro, M.; Lombez, L.; Braun, P.-F.; Marie, X.; Amand, T.; Gérard, J.-M. Linear and Dynamical Photoinduced Dichroisms of InAs/GaAs SelfAssembled Quantum Dots: Population Relaxation and Decoherence Measucrements. Phys. Rev. B: Condens. Matter Mater. Phys. 2006, 73, 085301.

(74) Gourdon, C.; Lavallard, P. Fine Structure of Heavy Excitons in GaAs/AlAs Superlattices. Phys. Rev. B: Condens. Matter Mater. Phys. 1992, 46, 4644-4650.

(75) Van der Poel, W. A. J. A.; Severens, A. L. G. J.; Foxon, C. T. Quantum Beats in the Exciton Emission of Type II GaAs/AlAs Quantum Wells. Opt. Commun. 1990, 76, 116-120.

(76) Tartakovskii, A. I.; Cahill, J.; Makhonin, M. N.; Whittaker, D. M.; Wells, J.-P. R.; Fox, A. M.; Mowbray, D. J.; Skolnick, M. S.; Groom, K. M.; Steer, M. J.; Hopkinson, M. Dynamics of Coherent and Incoherent Spin Polarizations in Ensembles of Quantum Dots. Phys. Rev. Lett. 2004, 93, 057401.

(77) Fu, M.; Tamarat, P.; Huang, H.; Even, J.; Rogach, A. L.; Lounis, B. Neutral and Charged Exciton Fine Structure in Single Lead Halide Perovskite Nanocrystals Revealed by Magneto-Optical Spectroscopy. Nano Lett. 2017, 17, 2895-2901.

(78) Ben Aich, R.; Saïdi, I.; Ben Radhia, S.; Boujdaria, K.; Barisien, T.; Legrand, L.; Bernardot, F.; Chamarro, M.; Testelin, C. BrightExciton Splittings in Inorganic Cesium Lead Halide Perovskite Nanocrystals. Phys. Rev. Appl. 2019, 11, 034042.

(79) Gupalov, S. V.; Ivchenko, E. L.; Kavokin, A. V. Fine Structure of Localized Exciton Levels in Quantum Wells. J. Exp. Theor. Phys. 1998, 86, 388-394.

(80) Hu, Z.; Singh, A.; Goupalov, S. V.; Hollingsworth, J. A.; Htoon, $\mathrm{H}$. Influence of Morphology on the Blinking Mechanisms and the Excitonic Fine Structure of Single Colloidal Nanoplatelets. Nanoscale 2018, 10, 22861-22870.

(81) Ivchenko, E. L.; Kaminskii, A. Y.; Aleiner, I. L. Exchange Splitting of Excitonic Levels in Types I and II Superlattices. J. Exp. Theor. Phys. 1993, 77, 609-616.

(82) Maialle, M. Z.; de Andrada e Silva, E. A.; Sham, L. J. Exciton Spin Dynamics in Quantum Wells. Phys. Rev. B: Condens. Matter Mater. Phys. 1993, 47, 15776-15788.

(83) Trif, M.; Simon, P.; Loss, D. Relaxation of Hole Spins in Quantum Dots via Two-Phonon Processes. Phys. Rev. Lett. 2009, 103, 106601 .

(84) Shamirzaev, T. S.; Rautert, J.; Yakovlev, D. R.; Debus, J.; Gornov, A. Y.; Glazov, M. M.; Ivchenko, E. L.; Bayer, M. Spin Dynamics and Magnetic Field Induced Polarization of Excitons in Ultrathin GaAs/AlAs Quantum Wells with Indirect Band Gap and Type-II Band Alignment. Phys. Rev. B: Condens. Matter Mater. Phys. 2017, 96, 035302. 